\title{
The lollipop graph is determined by its spectrum
}

\author{
R. Boulet, B. Jouve \\ Institut de Mathématiques \\ Université de Toulouse et CNRS (UMR 5219) \\ \{boulet, jouve\}@univ-tlse2.fr
}

Submitted: Feb 07, 2008; Accepted: May 20, 2008; Published: May 26, 2008

Mathematics Subject Classifications: 05C50, 68R10

\begin{abstract}
An even (resp. odd) lollipop is the coalescence of a cycle of even (resp. odd) length and a path with pendant vertex as distinguished vertex. It is known that the odd lollipop is determined by its spectrum and the question is asked by W. Haemers, X. Liu and Y. Zhang for the even lollipop. A private communication of Behruz Tayfeh-Rezaie pointed out that an even lollipop with a cycle of length at least 6 is determined by its spectrum but the result for lollipops with a cycle of length 4 is still unknown. We give an unified proof for lollipops with a cycle of length not equal to 4 , generalize it for lollipops with a cycle of length 4 and therefore answer the question. Our proof is essentially based on a method of counting closed walks.
\end{abstract}

\section{Contents}

1 Introduction $\quad 2$

2 Basic results and revisited proofs $\quad 3$

2.1 Counting the closed walks . . . . . . . . . . . . . . 3

2.2 Known results . . . . . . . . . . . . . . . . . . . . . 5

2.3 Bounds on eigenvalues . . . . . . . . . . . . . . . . . . . 7

2.4 There are no cospectral non-isomorphic lollipops: revisited proof . . . . . . 9

2.5 The lollipop $L(p, k), p \neq 4$, is determined by its spectrum . . . . . . . . . . 10

3 The lollipop $L(4, k)$ is determined by its spectrum $\quad \mathbf{1 1}$

3.1 Connectivity and case II . . . . . . . . . . . . . . . . . . 11

3.2 Our toolbox: some results on $L(4, k) \ldots \ldots \ldots \ldots$. . . . . . . . . . . 12

3.3 Case III: graphs with $n_{3}=n_{1}=3, n_{0}=0, c=0$ and $n_{2}=n-6 \ldots 14$

3.3.1 Unicyclic graphs with exactly three vertices of maximal degree 3 of which only one belongs to the cycle . . . . . . . . . . . 15 
3.3.2 Unicyclic graphs with exactly three vertices of maximum degree 3 of which exactly two belong to the cycle . . . . . . . . . 18

3.3.3 Unicyclic graphs with exactly three vertices of maximum degree 3, all of them belonging to the cycle . . . . . . . . . . 24

3.4 Case IV: Graphs with $n_{4}=1, n_{3}=0 n_{1}=2, n_{0}=0, c=0$ and $n_{2}=n-3 \quad 30$

\section{Conclusion}

A Appendix 32

A.1 Counting covering closed walks $\ldots \ldots \ldots \ldots \ldots \ldots \ldots$

A.2 Proof of theorem $17 \ldots \ldots \ldots \ldots \ldots \ldots$

A.3 Tables of some graphs eigenvalues . . . . . . . . . . . . . . 40

\section{Introduction}

Let $G$ be a simple graph with $n$ vertices and $A$ its adjacency matrix, $Q_{G}(X)$ denotes its characteristic polynomial and $\lambda_{1}(G) \geq \lambda_{2}(G) \geq \cdots \geq \lambda_{n}(G)$ the associated eigenvalues; $\lambda_{1}(G)$ is the spectral radius of $G$. It is known that some informations about the graph structure can be deduced from these eigenvalues such as the number of edges or the length of the shortest odd cycle; but the reverse question Which graphs are determined by their spectrum? (asked, among others, in [4]) is far from being solved; some partial results exist $[5,10,12]$ which contribute to answer this question.

Let us remind that the coalescence of two graphs $G_{1}$ with distinguished vertex $v_{1}$ and $G_{2}$ with distinguished vertex $v_{2}$, is formed by identifying vertices $v_{1}$ and $v_{2}$ that is, the vertices $v_{1}$ and $v_{2}$ are replaced by a single vertex $v$ adjacent to the same vertices in $G_{1}$ as $v_{1}$ and the same vertices in $G_{2}$ as $v_{2}$. If it is not necessary $v_{1}$ or $v_{2}$ may not be specified.

A lollipop $L(p, k)$ is the coalescence of a cycle $C_{p}$ with $p \geq 3$ vertices and a path $P_{k+1}$ with $k+1 \geq 2$ vertices with one of its vertex of degree one as distinguished vertex, figure 1 shows an example of a lollipop. The lollipop $L(p, 0)$ is $C_{p}$. An even (resp. odd) lollipop has a cycle of even (resp. odd) length. In this paper we shall show that the lollipop graph is determined by its spectrum, answering an open question asked in $[8,3]$ for even lollipop. It is known [8] that the odd lollipop is determined by its spectrum, but the proof given in $[8]$ cannot be generalized for even lollipops.

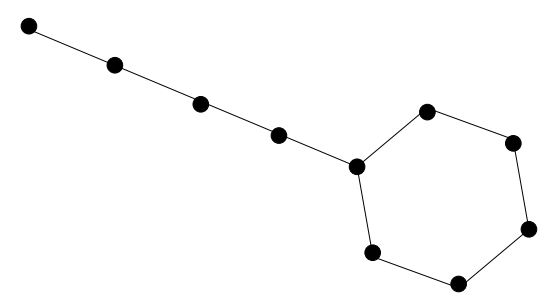

Figure 1: Lollipop L(6,4) 
We describe in section 2 some basic results of spectral graph theory we shall use in the following of the paper. We also explain the method we use to count closed walks in a graph and revisit the proof of a result about lollipops. We end this section with an unified proof of the fact that a lollipop $L(p, k), p \neq 4$ is determined by its spectrum. The main section of the paper (section 3 ) shows that the lollipop $L(4, k)$ is determined by its spectrum; in subsection 3.1 we show that a graph cospectral with a $L(4, k)$ is connected, then we expose in subsection 3.2 the toolbox we use to show that the graphs considered in subsections 3.3 and 3.4 are not cospectral with a lollipop $L(4, k)$.

To fix notations, the disjoint union of two graphs $G$ and $H$ is noted $G \cup H$.

As defined in [12] a T-shape tree $S_{a, b, c}(a, b, c>0)$ is a tree with one and only one vertex $v$ of degree 3 such that $S_{a, b, c} \backslash\{v\}=P_{a} \cup P_{b} \cup P_{c}$. We extend this notation for all $b, c \in \mathbb{N}$ by $S_{0, b, c}=P_{b+c+1}$.

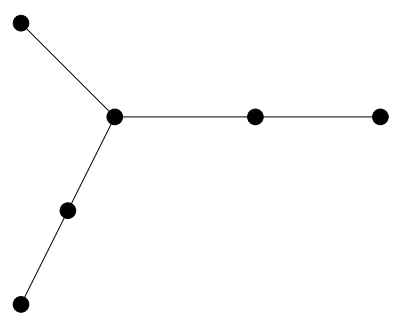

Figure 2: $S_{1,2,2}$

By $S_{n-1}$ we denote the star with $n$ vertices and by $T_{n}$ the tree with $n$ vertices drawn on figure 3.

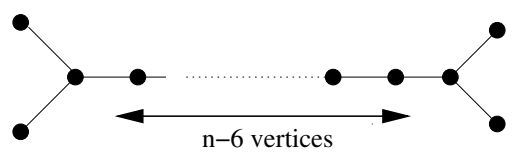

Figure 3: $T_{n}$

Finally let $d(u, v)$ be the distance (the length of a shortest path) between two vertices $u$ and $v$ and $\delta(v)$ the degree of a vertex $v$.

\section{Basic results and revisited proofs}

\subsection{Counting the closed walks}

It is a classical result that the number of closed walks of length $k \geq 2$ is $\sum_{i} \lambda_{i}^{k}$

We describe here a method to count the number of closed walks of a given length within a graph. 
Let $M$ be a graph, a $k$-covering closed walk in $M$ is a closed walk of length $k$ in $M$ running through all the edges at least once. Let $G$ be a graph, $M(G)$ denotes the set of all distinct subgraphs (not necessarily induced) of $G$ isomorphic to $M$ and $|M(G)|$ is the number of elements of $M(G)$. According to that point of view, $M$ may be called a motif (or a pattern). The number of $k$-covering closed walks in a motif $M$ is denoted by $w_{k}(M)$ and we define the set $\mathcal{M}_{k}=\left\{M, w_{k}(M)>0\right\}$ and we note that the set $\left\{M \in \mathcal{M}_{k},|M(G)|>0\right\}$ is finite (if $G$ is a finite graph).

As a consequence, the number of closed walks of length $k$ in $G$ is:

$$
\sum_{i} \lambda_{i}^{k}=\sum_{M \in \mathcal{M}_{k}} w_{k}(M)|M(G)|
$$

In practice, there are at least two methods to determine $w_{k}(M)$ : on one hand a combinatorial way which counts the number of covering closed walks of length $k$ in $M$, on the other hand an algebraic method which uses the following straightforward formula:

$$
w_{k}(M)=\sum_{\lambda_{i} \in \operatorname{Sp}(M)} \lambda_{i}^{k}-\sum_{M^{\prime} \in \mathcal{M}_{k}, M^{\prime} \neq M} w_{k}\left(M^{\prime}\right)\left|M^{\prime}(M)\right|
$$

where $\operatorname{Sp}(M)$ denotes the spectrum of the adjacency matrix of $M$.

Using equation (1) and table 5 in appendix, we have the following proposition:

Proposition 1. i) If $G$ is a graph without triangles and $C_{5}$ then:

$$
\begin{aligned}
\sum_{i} \lambda_{i}^{6}= & 12\left|C_{6}(G)\right|+2\left|P_{2}(G)\right|+12\left|P_{3}(G)\right|+6\left|P_{4}(G)\right|+12\left|S_{1,1,1}(G)\right| \\
& +48\left|C_{4}(G)\right|+12|L(4,1)(G)|
\end{aligned}
$$

ii) If $G$ is a graph without $C_{p}, p \in\{3,5,6,7\}$ and of maximal degree 3 then:

$$
\begin{aligned}
\sum_{i} \lambda_{i}^{8}= & 2\left|P_{2}(G)\right|+28\left|P_{3}(G)\right|+32\left|P_{4}(G)\right|+8\left|P_{5}(G)\right| \\
& +72\left|S_{1,1,1}(G)\right|+16\left|S_{1,1,2}(G)\right|+264\left|C_{4}(G)\right| \\
& +112|L(4,1)(G)|+16|L(4,2)(G)|+16\left|C_{8}(G)\right|
\end{aligned}
$$

iii) If $G$ is a graph without $C_{p}, p \in\{3,5,6,7,8,9\}$, of maximal degree 3 and such that $\delta(u)=\delta(v)=3, u \neq v \Rightarrow d(u, v)>1$, then:

$$
\begin{aligned}
\sum_{i} \lambda_{i}^{10}= & 2\left|P_{2}(G)\right|+60\left|P_{3}(G)\right|+120\left|P_{4}(G)\right|+60\left|P_{5}(G)\right|+10\left|P_{6}(G)\right| \\
& +300\left|S_{1,1,1}(G)\right|+140\left|S_{1,1,2}(G)\right|+20\left|S_{1,2,2}(G)\right| \\
& +20\left|S_{1,1,3}(G)\right|+1320\left|C_{4}(G)\right|+840|L(4,1)(G)| \\
& +180|L(4,2)(G)|+20|L(4,3)(G)|+20\left|C_{10}(G)\right|
\end{aligned}
$$


In this paper we shall have to count all the $|M(G)|, M \in \mathcal{M}_{i}$ of a given unicyclic graph $G$. For that aim we describe here the steps of the process we follow to count the $P_{k}(G)$ which are the only motifs hard to count. Let $p$ be the length of the cycle of $G$.

ALGORITHM to count $P_{k}(G)$ :

set $H=G$

set $\left|P_{k}(G)\right|=0$.

while there exists a pendant vertex $u$ in $H$ do

count the number $q$ of paths $P_{k}$ of $H$ containing $u$

let $\left|P_{k}(G)\right|=\left|P_{k}(G)\right|+q$

let $H=H \backslash\{u\}$

end while

if $p \geq k$ then

$\left|P_{k}(G)\right|=\left|P_{k}(G)\right|+p$

end if

return $\left|P_{k}(G)\right|$

\subsection{Known results}

Proposition 2. [2] Let $G$ be a graph with $n$ vertices and $m$ edges and let $\lambda_{i}$ its associated eigenvalues. We have: $\sum_{i} \lambda_{i}^{4}=8\left|C_{4}(G)\right|+2 m+4\left|P_{3}(G)\right|$. Let $n_{k}$ be the number of vertices of degree $k$ in $G$, we have:

$$
\sum_{i} \lambda_{i}^{4}=8 c_{4}+\sum_{k} k n_{k}+4 \sum_{k \geq 2} \frac{k(k-1)}{2} n_{k}
$$

The following result relates the coefficients of the characteristic polynomial of a graph with structural properties of this graph:

Theorem 1. [1] Let $Q_{G}(X)=X^{n}+a_{1} X^{n-1}+a_{2} X^{n-2}+\ldots+a_{n}$ be the characteristic polynomial of a graph $G$. We call an "elementary figure" the graph $P_{2}$ or the graphs $C_{q}, q>0$. We call a "basic figure" $U$ every graph all of whose components are elementary figures. Let $p(U)$ be the number of connected components of $U$ and $c(U)$ the number of cycles in $U$. We note $\mathcal{U}_{i}$ the set of basic figures with $i$ vertices. Then

$$
a_{i}=\sum_{U \in \mathcal{U}_{i}}(-1)^{p(U)} 2^{c(U)}, i=1,2, \ldots, n
$$

It follows this theorem:

Theorem 2. [1] Let $Q_{G}(X)=X^{n}+a_{1} X^{n-1}+a_{2} X^{n-2}+\ldots+a_{n}$ be the characteristic polynomial of a graph $G$. The length of the shortest odd cycle in $G$ is given by the smallest odd index $p$ such that $a_{p} \neq 0$ and the value of $a_{p}$ gives the number of $p$-cycles in $G$. 
It ensues that a bipartite graph ( $i e$ a graph with no odd cycles) cannot be cospectral with a non-bipartite graph.

The following result is useful at many time in the paper, for instance to find bounds on eigenvalues:

Theorem 3 (Interlacing theorem). [7] Let $G$ be a graph with $n$ vertices and associated eigenvalues $\lambda_{1} \geq \lambda_{2} \geq \ldots \geq \lambda_{n}$ and let $H$ be an induced subgraph of $G$ with $m$ vertices and associates eigenvalues $\mu_{1} \geq \mu_{2} \geq \ldots \geq \mu_{m}$. Then for $i=1, \ldots, m, \lambda_{n-m+i} \leq \mu_{i} \leq \lambda_{i}$.

The next theorems give a way to compute the characteristic polynomial of a graph by deleting a vertex or an edge:

Theorem 4. [1] Let $G$ be a graph obtained by joining by an edge a vertex $x$ of a graph $G_{1}$ and a vertex $y$ of a graph $G_{2}$. Then

$$
Q_{G}(X)=Q_{G_{1}}(X) Q_{G_{2}}(X)-Q_{G_{1} \backslash x}(X) Q_{G_{2} \backslash y}(X)
$$

Theorem 5. [1] Let $G$ be a graph and $x$ a vertex of $G$, then:

$$
Q_{G}(X)=X Q_{G \backslash x}(X)-\sum_{y \sim x} Q_{G \backslash\{x, y\}}(X)-2 \sum_{C, x \in C} Q_{G \backslash C}(X)
$$

where $y \sim x$ means that $y x$ is an edge of $G$ and the second sum is on the set of the cycles $C$ containing $x$.

Theorem 6. [1] Let $G$ be a graph and $x$ a pendant vertex of $G$. Then:

$$
Q_{G}(X)=X Q_{G \backslash x}(X)-Q_{G \backslash\{x, y\}}(X)
$$

where $y$ is the neighbor of $x$.

Property 1. We have the following equalities:

$Q_{C_{p}}(X)=X Q_{P_{p-1}}(X)-2 Q_{P_{p-2}}(X)-2$

$Q_{P_{p}}(X)=X Q_{P_{p-1}}(X)-Q_{P_{p-2}}(X)$

Proof. A direct consequence of theorems 4 and 6 .

The following theorem relates the behavior of the spectral radius of a graph by subdividing an edge. An internal path of a graph $G$ is an elementary path $x_{0} x_{1} \cdots x_{k}(i e$ $x_{i} \neq x_{j}$ for all $i \neq j$ but eventually $x_{0}=x_{k}$ ) of $G$ with $\delta\left(x_{0}\right)>2, \delta\left(x_{k}\right)>2, \delta\left(x_{i}\right)=2$ for all other $i$ 's.

Theorem 7. [11, 9] Let xy be an edge of a connected graph $G$ not belonging to an internal path, then the spectral radius strictly increases by subdividing xy.

Let $x y$ be an edge of a connected graph $G \neq T_{n}$ belonging to an internal path, then the spectral radius strictly decreases by subdividing $x y$.

Theorem 8. [6] Let $G$ be a graph with maximal degree $\delta_{M}$, then $\lambda_{1}(G) \geq \sqrt{\delta_{M}}$.

Let $B(p, q)$ be the coalescence of two cycles $C_{p}$ and $C_{q}$ (see figure 4 for an example).

Theorem 9. [11] For $p \geq 3, q \geq 3, \lambda_{1}(B(p, q))>\frac{4}{\sqrt{3}}>\sqrt{5}$. 


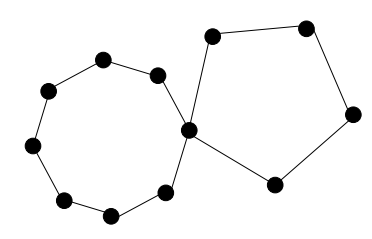

Figure 4: $\mathrm{B}(8,5)$

\subsection{Bounds on eigenvalues}

Theorem 7 gives the following corollaries:

Corollary 1. $\lambda_{1}(L(p, k))>\lambda_{1}(L(p+1, k))$.

Corollary 2. $\lambda_{1}(L(p, k))<\lambda_{1}(L(p, k+1))$.

Given $p \geq 3, q \geq 3$, let $H(p, q)$ be the coalescence of $C_{p}$ and $L(q, 1)$ with the pendant vertex as distinguished vertex (see figure 5 for an example).

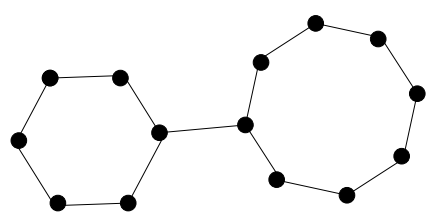

Figure 5: $\mathrm{H}(6,8)$

Theorem 10. $\lambda_{1}(H(p, q))>\sqrt{5}$.

Proof. Without loss of generality we suppose that $p \geq q$. According to theorem 7 we have $\lambda_{1}(H(p, q)) \geq \lambda_{1}(H(p, p))$ so it is sufficient to prove the theorem for $H(p, p)$. As $\lim _{x \rightarrow+\infty} Q_{H(p, q)}(x)=+\infty$ it is sufficient to prove that $Q_{H(p, p)}(\sqrt{5})<0$

Theorem 4 gives:

$$
\begin{gathered}
Q_{H(p, p)}(X)=Q_{C_{p}}(X) Q_{C_{p}}(X)-Q_{P_{p-1}}(X) Q_{P_{p-1}}(X) \\
Q_{H(p, p)}(X)=\left[Q_{C_{p}}(X)\right]^{2}-\left[Q_{P_{p-1}}(X)\right]^{2}
\end{gathered}
$$

and by property 1 we have:

$$
Q_{H(p, p)}(X)=\left[X Q_{P_{p-1}}(X)-2 Q_{P_{p-2}}(X)-2\right]^{2}-\left[Q_{P_{p-1}}(X)\right]^{2}
$$

Let $\left(u_{n}\right)_{n \in \mathbb{N}}$ be the sequence defined by $u_{n}=Q_{P_{n}}(\sqrt{5})$. We have (property 1$): u_{n}=$ $\sqrt{5} u_{n-1}-u_{n-2}$. Since $u_{1}=\sqrt{2}$ and $u_{2}=4$ then $u_{n}=\beta_{1}^{n+1}-\beta_{2}^{n+1}$ where $\beta_{1}=\frac{\sqrt{5}+1}{2}$ and $\beta_{2}=\frac{\sqrt{5}-1}{2}$. 


$$
\begin{aligned}
Q_{H(p, p)}(\sqrt{5}) & =\left[\sqrt{5} u_{p-1}-2 u_{p-2}-2\right]^{2}-\left(u_{p-1}\right)^{2} \\
& =\left[(\sqrt{5}+1) \beta_{1}^{p-1}-2\right]\left[(\sqrt{5}-1) \beta_{2}^{p-1}-2\right]
\end{aligned}
$$

We have $\left[(\sqrt{5}+1) \beta_{1}^{p-1}-2\right]>0$ and $\left[(\sqrt{5}-1) \beta_{2}^{p-1}-2\right]<0$ so $Q_{H(p, p)}(\sqrt{5})<0$.

Theorem 11. For $k \neq 0$ we have $\lambda_{1}(L(p, k))>2$ and $\lambda_{2}(L(p, k))<2$.

\section{Proof.}

$\lambda_{1}(L(p, k))>2$ : the spectral radius of a cycle is 2 and a cycle is an induced subgraph of $L(p, k)$ so by the interlacing theorem we have $\lambda_{1}(L(p, k)) \geq 2$. It remains to show that $\lambda_{1}(L(p, k)) \neq 2$. By theorem 4 we have $Q_{L(p, k)}(2)=Q_{C_{p}}(2) Q_{P_{k}}(2)-Q_{P_{p-1}}(2) Q_{P_{k-1}}(2)=$ $-Q_{P_{p-1}}(2) Q_{P_{k-1}}(2) \neq 0$ (because the spectral radius of a path is strictly less than 2 ).

$\lambda_{2}(L(p, k))<2$ : the path $P_{p+k-1}$ is an induced subgraph of $L(p, k)$ so by the interlacing theorem we have $\lambda_{2}(L(p, k)) \leq \lambda_{1}\left(P_{p+k-1}\right)<2$.

Theorem 12. i) We have, for $p \geq 3, \lambda_{1}(L(p, k))<\sqrt{5} \approx 2.2361$.

ii) We have, for $p \geq 4, \lambda_{1}(L(p, k))<\sqrt{2+2 \sqrt{2}} \approx 2.1974$.

Proof. i) By corollary 1 we have $\lambda_{1}(L(p, k)) \leq \lambda_{1}(L(3, k))$ so it is sufficient to prove the theorem for $p=3$. For $k=0, \lambda_{1}(L(3,0))=2<\sqrt{5}$. We now assume that $k>0$. Using theorem 4 and $Q_{C_{3}}(X)=(X+1)^{2}(X-2)$ we have:

$$
Q_{L(3, k)}(X)=(X+1)^{2}(X-2) Q_{P_{k}}(X)-(X-1)(X+1) Q_{P_{k-1}}(X)
$$

and

$$
Q_{L(3, k)}(\sqrt{5})=(2 \sqrt{5}-2) Q_{P_{k}}(\sqrt{5})-4 Q_{P_{k-1}}(\sqrt{5})
$$

Let us suppose that $Q_{L(3, k)}(\sqrt{5})>0$.

We have

$$
Q_{L(3, k+1)}(\sqrt{5})=(2 \sqrt{5}-2) Q_{P_{k+1}}(\sqrt{5})-4 Q_{P_{k}}(\sqrt{5})
$$

but

$$
Q_{P_{k+1}}(\sqrt{5})=\sqrt{5} Q_{P_{k}}(\sqrt{5})-Q_{P_{k-1}}(\sqrt{5})
$$

so

$$
Q_{L(3, k+1)}(\sqrt{5})=\frac{2 \sqrt{5}-2}{4}\left((2 \sqrt{5}-2) Q_{P_{k}}(\sqrt{5})-4 Q_{P_{k-1}}(\sqrt{5})\right)
$$

and by induction on $k \geq 1$ we have $Q_{L(3, k+1)}(\sqrt{5})>0$.

Since the polynomial $Q_{L(3, k)}$ has one and only one root in $] 2,+\infty[$ (theorem 11) then $Q_{L(3, k)}(2)<0$ and $Q_{L(3, k)}(\sqrt{5})>0$ implies that $\lambda_{1}(L(3, k))<\sqrt{5}$. 
ii) The proof of ii) is led in the same way. Let $\alpha=\sqrt{2+2 \sqrt{2}}$. We have:

$$
Q_{L(4, k)}(X)=X^{2}\left(X^{2}-4\right) Q_{P_{k}}(X)-X\left(X^{2}-2\right) Q_{P_{k-1}}(X)
$$

and

$$
Q_{L(4, k)}(\alpha)=4 Q_{P_{k}}(\alpha)-2 \sqrt{2} \alpha Q_{P_{k-1}}(\alpha)
$$

Let us suppose that $Q_{L(4, k)}(\alpha)>0$. As $Q_{P_{k+1}}(\alpha)=\alpha Q_{P_{k}}(\alpha)-Q_{P_{k-1}}(\alpha)$ we have

$$
Q_{L(4, k+1)}(\alpha)=\frac{2-\sqrt{2}}{2} \alpha\left(4 Q_{P_{k}}(\alpha)-2 \sqrt{2} \alpha Q_{P_{k-1}}(\alpha)\right)
$$

and by induction on $k \geq 1$ we have $Q_{L(4, k+1)}(\alpha)>0$.

Theorem 13. Let $G$ be a graph cospectral with $L(p, k)$, then

$$
\max \{\delta(v), v \in V(G)\} \leq 4 .
$$

Proof. A direct consequence of theorems 12 and 8 .

Theorem 14. Let $G$ be a graph cospectral with a lollipop. Then, for $p \geq 3$ and $q \geq 3$, $C_{p} \cup C_{q}$ or $H(p, q)$ or $B(p, q)$ cannot be induced subgraphs of $G$

Proof. If $C_{p} \cup C_{q}$ is an induced subgraph of $G$ then as $\lambda_{2}\left(C_{p} \cup C_{q}\right)=2$ by interlacing theorem we get $\lambda_{2}(G) \geq 2$, impossible by theorem 11 .

$H(p, q)$ or $B(p, q)$ cannot be induced subgraphs of $G$ because $\lambda_{1}(G)<\sqrt{5}$ (theorem 12) and $\lambda_{1}(H(p, q))>\sqrt{5}$ (theorem 10), $\lambda_{1}(B(p, q))>\sqrt{5}$ (theorem 9$)$.

\subsection{There are no cospectral non-isomorphic lollipops: revisited proof}

In [8] it is proved that two cospectral lollipops are isomorphic. We revisit here this result in a shortest proof using closed walks.

Theorem 15. There are no cospectral non-isomorphic lollipops.

Proof. Let $L(p, k)$ and $L\left(p^{\prime}, k^{\prime}\right)$ with $n=p+k=p^{\prime}+k^{\prime}$ and $p<p^{\prime}$ be two non isomorphic lollipops. To show that they have different spectra we show that there are less closed walks of length $p$ in $L\left(p^{\prime}, k^{\prime}\right)$ than in $L(p, k)$.

Let $e$ (resp. $\left.e^{\prime}\right)$ be an edge of the cycle of $L(p, k)$ (resp. $\left.L\left(p^{\prime}, k^{\prime}\right)\right)$ incident to the vertex of degree $3, \mathcal{W}\left(\operatorname{resp} \mathcal{W}^{\prime}\right)$ the set of closed walks of length $p$ of $L(p, k)\left(\operatorname{resp} . L\left(p^{\prime}, k^{\prime}\right)\right), \hat{\mathcal{W}}$ 
(resp $\left.\hat{\mathcal{W}}^{\prime}\right)$ the set of closed walks of length $p$ of $L(p, k)$ (resp. $L\left(p^{\prime}, k^{\prime}\right)$ ) not containing $e$ (resp. $\left.e^{\prime}\right)$ and $\tilde{\mathcal{W}}\left(\operatorname{resp} \tilde{\mathcal{W}}^{\prime}\right)$ the set of closed walks of length $p$ of $L(p, k)$ (resp. $\left.L\left(p^{\prime}, k^{\prime}\right)\right)$ containing $e$ (resp. $\left.e^{\prime}\right)$.

We have: $|\mathcal{W}|=|\hat{\mathcal{W}}|+|\tilde{\mathcal{W}}|$ (resp. $\left.\left|\mathcal{W}^{\prime}\right|=\left|\hat{\mathcal{W}}^{\prime}\right|+\left|\tilde{\mathcal{W}}^{\prime}\right|\right)$. It's obvious that $|\hat{\mathcal{W}}|=\left|\hat{\mathcal{W}}^{\prime}\right|$ because $L(p, k) \backslash\{e\}=L\left(p^{\prime}, k^{\prime}\right) \backslash\left\{e^{\prime}\right\}=P_{n}$. We are going to show that $|\tilde{\mathcal{W}}|<\left|\tilde{\mathcal{W}}^{\prime}\right|$ by the following equation:

$$
|\tilde{\mathcal{W}}|=\sum_{M \in \mathcal{M}_{p}, e \in E(M)} w_{p}(M)|M(G)|
$$

where $E(M)$ is the set of the edges of $M$.

We denote by $M^{e}$ a motif $M$ containing $e$. The motifs containing $e$ (resp $e^{\prime}$ ) with at least one $p$-covering closed walk are exactly:

- the $P_{i}^{\prime}$ 's for $2 \leq i \leq \frac{p}{2}+1$ (if $p$ is even) and we have $\left|P_{i}^{e^{\prime}}\left(L\left(p^{\prime}, k^{\prime}\right)\right)\right| \leq\left|P_{i}^{e}(L(p, k))\right|$.

- the $S_{a, b, c}$ 's with $a+b+c \leq \frac{p}{2}$ (if $p$ is even) and we have $\left|S_{a, b, c}^{e^{\prime}}\left(L\left(p^{\prime}, k^{\prime}\right)\right)\right| \leq\left|S_{a, b, c}^{e}(L(p, k))\right|$.

- the $C_{p}$ 's and $0=\left|C_{p}^{e^{\prime}}\left(L\left(p^{\prime}, k^{\prime}\right)\right)\right|<\left|C_{p}^{e}(L(p, k))\right|=1$.

So, $|\tilde{\mathcal{W}}|<\left|\tilde{\mathcal{W}}^{\prime}\right|$ and $|\mathcal{W}|<\left|\mathcal{W}^{\prime}\right|$ which concludes the proof.

\subsection{The lollipop $L(p, k), p \neq 4$, is determined by its spectrum}

This result mentionned in [8,3] for even lollipops with $p \geq 6$ as a private communication of Behruz Tayfeh-Rezaie is true for all lollipops $L(p, k)$ with $p \neq 4$. The aim of the proof is to determine the degree distribution. We already know that there are no vertices of degree greater or equal than 5 (theorem 13).

Theorem 16. Let $G$ be a graph cospectral with $L(p, k), p \neq 4$. Then $G$ is isomorphic to $L(p, k)$.

Proof. Let $n_{i}$ be the number of vertices of degree $i$ for $i \in\{0,1,2,3,4\}$. We have $n=n_{0}+n_{1}+n_{2}+n_{3}+n_{4}$ and $2 n=n_{1}+2 n_{2}+3 n_{3}+4 n_{4}$ (the sum of the degrees is twice the number of edges), so $2 n_{0}+n_{1}=n_{3}+2 n_{4}$.

Let $c=\left|C_{4}(G)\right|$, we have by proposition $2, \sum_{\lambda_{i} \in \operatorname{Sp}(G)} \lambda_{i}^{4}=8 c+2 m+4\left(n_{2}+3 n_{3}+6 n_{4}\right)$. As $\sum_{\lambda_{i} \in \operatorname{Sp}(G)} \lambda_{i}^{4}=\sum_{\lambda_{i} \in \operatorname{Sp}(L(p, k))} \lambda_{i}^{4}$ we get $5 n_{4}+2 n_{3}-n_{0}-n_{1}+2 c=1$ and, using $2 n_{0}+n_{1}=n_{3}+2 n_{4}$, we have $3 n_{4}+n_{3}+n_{0}+2 c=1$.

So $n_{4}=0$ and $c=0$. If $n_{3}=0$ then $n_{0}=1$ which contradicts $2 n_{0}+n_{1}=n_{3}+2 n_{4}$. So $n_{3}=1$ and $n_{0}=0$. We deduce $n_{1}=1$ and $n_{2}=n-2$.

As the sum of the degrees of a graph is even, the vertex of degree 1 and the vertex of degree 3 belongs to the same connected component. If $G$ is not connected there is a 2regular connected component ( $i e$ a cycle) which is impossible ( 2 is not an eigenvalue of $G$ ). As a result, $G$ is a connected graph with degree distribution equal to $(1,2,2,2, \ldots, 2,2,3)$, so $G$ is a lollipop and, by theorem $15, G$ is isomorphic to $L(p, k)$. 


\section{The lollipop $L(4, k)$ is determined by its spectrum}

Let $G$ be a graph cospectral with $L(4, k)$, let $n_{i}, i \in\{0,1,2,3,4\}$, be the number of vertices of degree $i$ of $G$ and let $c=\left|C_{4}(G)\right|$. If we use the same method as previously to prove

that a lollipop $L(4, k)$ is determined by its spectrum, we obtain $\sum_{\lambda_{i} \in \operatorname{Sp}(L(4, k))} \lambda_{i}^{4}=6 n+12$ and we have to solve

$$
3 n_{4}+n_{3}+n_{0}+2 c=3
$$

Noting that $2 n_{0}+n_{1}=n_{3}+2 n_{4}$, we have to consider the following cases:

- case I: $n_{4}=0, n_{3}=1, n_{0}=0, c=1$ and consequently $n_{1}=1, n_{2}=n-2$.

- case II: $n_{4}=0, n_{3}=2, n_{0}=1, c=0$ and consequently $n_{1}=0, n_{2}=n-3$.

- case III: $n_{4}=0, n_{3}=3, n_{0}=0, c=0$ and consequently $n_{1}=3, n_{2}=n-6$.

- case IV: $n_{4}=1, n_{3}=0, n_{0}=0, c=0$ and consequently $n_{1}=2, n_{2}=n-3$.

The first case implies that the graph is $L(4, k)$, it remains to show that the last three cases are impossible, this is done in the next subsections. In order to simplify the enumeration of graphs in the cases II, III and IV, we before establish the connectivity of a graph cospectral with $L(4, k)$.

\subsection{Connectivity and case II}

In order to simplify the study of these three cases we show in this section that a graph cospectral with a $L(4, k)$ is connected. As noticed in [8] this is a difficult problem and to lighten this section some technical proofs have been detailed in appendix.

Using results of section 2.2 we easily obtain the following property:

Property 2. $\forall a, b, c \in \mathbb{N}, k \in \mathbb{N}^{*}, p>2: Q_{C_{p}}(2)=0, Q_{P_{k}}(2)=k+1, Q_{S_{a, b, c}}(2)=$ $a+b+c+2-a b c, Q_{S_{1,1, a}}(2)=4$.

Let $P\left(p_{1}, p_{2}, p_{3}\right)$ be the graph obtained by identifying the three pendant vertices of $S_{p_{1}+1, p_{2}+1, p_{3}+1}$ (an example is given in figure 6 ).

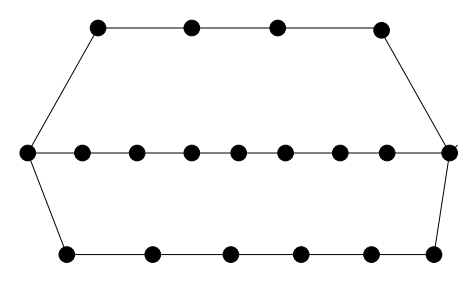

Figure 6: $\mathrm{P}(4,7,6)$ 
Theorem 17. The graph $P\left(p_{1}, p_{2}, p_{3}\right)$ cannot be an induced subgraph of a graph $G$ cospectral with a lollipop $L(4, k)$.

Proof. Sketch of the proof:

We first show that for some values of $p_{1}, p_{2}$ and $p_{3}$ we have $\lambda_{1}\left(P\left(p_{1}, p_{2}, p_{3}\right)\right)>$ $\sqrt{2+2 \sqrt{2}}$ and in these cases $P\left(p_{1}, p_{2}, p_{3}\right)$ cannot be an induced subgraph of G.

For the others cases we compute $Q_{P\left(p_{1}, p_{2}, p_{3}\right)}(2)$.

- if $Q_{P\left(p_{1}, p_{2}, p_{3}\right)}(2) \geq 0$ then $P\left(p_{1}, p_{2}, p_{3}\right)$ and a fortiori $G$ (interlacing theorem) possesses two eigenvalues greater than 2 which contradicts that $G$ is cospectral with a lollipop (theorem 11).

- if $Q_{P\left(p_{1}, p_{2}, p_{3}\right)}(2)<0$ then we show that $P\left(p_{1}, p_{2}, p_{3}\right)$ cannot be a connected component of $G$ so there is a vertex $x$ not in $P\left(p_{1}, p_{2}, p_{3}\right)$ adjacent to a vertex $y$ of $P\left(p_{1}, p_{2}, p_{3}\right)$ and we prove that this graph so constructed cannot be an induced subgraph of $G$.

A detailed proof is given in appendix A.

Theorem 18. Let $G$ be a graph cospectral with a lollipop $L(4, k)$. Then $G$ is connected.

Proof. The graph $G$ has as many edges as vertices, so if $G$ is not connected, it possesses at least two cycles. The subgraph induced by the two cycles of minimal length is $C_{a} \cup C_{b}, B(a, b), H(a, b)$ or $P\left(p_{1}, p_{2}, p_{3}\right)$ but this is impossible (theorems 14 and 17).

Corollary 3. A graph cospectral with a lollipop $L(4, k)$ is unicyclic.

This section solves the case II:

Corollary 4. There are no graphs cospectral with $L(4, k)$ and with $n_{3}=2, n_{1}=0, n_{0}=1$, $c=0$ and $n_{2}=n-3$.

\subsection{Our toolbox: some results on $L(4, k)$}

In the following we are going to prove that $L(4, k)$ is not cospectral with the unicyclic graphs of cases III and IV. For that purpose we use several tools detailed in this section: counting closed walks of length 6,8 or 10 , evaluating the characteristic polynomial in 1 or 2, using the fact that a lollipop has only one eigenvalue greater than 2 . 
Proposition 3. i) For $L(4, k), k>1$ we have:

$$
\sum_{i} \lambda_{i}^{6}=20 n+96
$$

ii) For $L(4, k), k>2$ we have:

$$
\sum_{i} \lambda_{i}^{8}=70 n+596
$$

iii) For $L(4, k), k>3$ we have

$$
\sum_{i} \lambda_{i}^{10}=252 n+3360
$$

Proof. Counting closed walks, we check that

i) For $k>1,\left|P_{2}(L(4, k))\right|=n,\left|P_{3}(L(4, k))\right|=n+1,\left|P_{4}(L(4, k))\right|=n+2,\left|C_{4}(L(4, k))\right|=$ $1,|L(4,1)(L(4, k))|=1$.

ii) Moreover, for $k>2,\left|P_{5}(L(4, k))\right|=n-1,\left|S_{1,1,1}(L(4, k))\right|=3,\left|S_{1,1,2}(L(4, k))\right|=3$, $|L(4,2)(L(4, k))|=1$.

iii) Moreover, for $k>3,\left|P_{6}(L(4, k))\right|=n-2,\left|S_{1,2,2}(L(4, k))\right|=2,\left|S_{1,1,3}(L(4, k))\right|=1$, $|L(4,2)(L(4, k))|=1,|L(4,3)(L(4, k))|=1$

and apply proposition 1 .

Property 3. We have $Q_{P_{p}}(1)=Q_{P_{\bar{p}}}(1)$ and $Q_{C_{p}}(1)=Q_{C_{\bar{p}}}(1)$ where $\bar{p}$ is $p$ modulo 6 and:

$$
\begin{array}{cc}
Q_{P_{\overline{0}}}(1)=1 & Q_{C_{\overline{0}}}(1)=0 \\
Q_{P_{\overline{1}}}(1)=1 & Q_{C_{\overline{1}}}(1)=-1 \\
Q_{P_{\overline{2}}}(1)=0 & Q_{C_{\overline{2}}}(1)=-3 \\
Q_{P_{\overline{3}}}(1)=-1 & Q_{C_{\overline{3}}}(1)=-4 \\
Q_{P_{\overline{4}}}(1)=-1 & Q_{C_{\overline{4}}}(1)=-3 \\
Q_{P_{\overline{5}}}(1)=0 & Q_{C_{\overline{5}}}(1)=-1
\end{array}
$$

Proof. According to property $1, Q_{P_{p}}(1)=Q_{P_{p-1}}(1)-Q_{P_{p-2}}(1)=-Q_{P_{p-3}}(1)=$ $Q_{P_{p-6}}(1)$ and $Q_{C_{p}}(1)=Q_{P_{p-1}}(1)-2 Q_{P_{p-2}}(1)-2$. Then we can easily compute $Q_{P_{i}}$ and $Q_{C_{i}}$ for $0 \leq i \leq 5$.

Property 4. We have:

$$
Q_{P_{k}}(0)=\left\{\begin{array}{c}
(-1)^{\frac{k}{2}} \text { if } k \text { is even } \\
0 \text { if } k \text { is odd }
\end{array}\right.
$$

and if $k$ is odd we have $R(0)=(-1)^{\frac{k-1}{2}} \frac{k+1}{2}$ where $R(X)=\frac{Q_{P_{k}}(X)}{X}$. 
Proof. Proofs by induction with the relation $Q_{P_{k}}(X)=X Q_{P_{k-1}}(X)-Q_{P_{k-2}}(X)$.

Proposition 4. We have:

$$
Q_{L(4, k)}(1)=\left\{\begin{array}{l}
1 \text { if } n \equiv 0[6] \\
3 \text { if } n \equiv 1[6] \\
2 \text { if } n \equiv 2[6] \\
-1 \text { if } n \equiv 3[6] \\
-3 \text { if } n \equiv 4[6] \\
-2 \text { if } n \equiv 5[6]
\end{array}\right.
$$

Proof. Theorem 4 gives $Q_{L(4, k)}(X)=Q_{C_{4}}(X) Q_{P_{k}}(X)-Q_{P_{3}}(X) Q_{P_{k-1}}(X)$ so $Q_{L(4, k)}(1)=-3 Q_{P_{k}}(1)+Q_{P_{k-1}}(1)$ and we conclude with property 3 .

Proposition 5. $Q_{L(4, k)}(2)=-4 n+16$.

Proof. $\quad Q_{L(4, k)}(X)=Q_{C_{4}}(X) Q_{P_{k}}(X)-Q_{P_{3}}(X) Q_{P_{k-1}}(X)$ and with property 2 we have $Q_{L(4, k)}(2)=-4 k=-4 n+16$.

Remark This proposition can be generalized for all lollipops: $Q_{L(p, k)}(2)=-p k$.

Proposition 6. If $n=4+k$ is even then 0 is an eigenvalue of $L(4, k)$ with multiplicity 2 and $R(0)=(-1)^{\frac{k}{2}+1} n$ where $R(X)=\frac{Q_{L(4, k)}(X)}{X^{2}}$.

Proof. Since $Q_{L(4, k)}(X)=Q_{C_{4}}(X) Q_{P_{k}}(X)-Q_{P_{3}}(X) Q_{P_{k-1}}(X)$ we have $R(X)=$ $\left(X^{2}-4\right) Q_{P_{k}}(X)-\left(X^{2}-2\right) \frac{Q_{P_{k-1}}(X)}{X}$ and property 4 gives the result.

\subsection{Case III: graphs with $n_{3}=n_{1}=3, n_{0}=0, c=0$ and $n_{2}=n-6$}

Let $G$ be a graph cospectral with $L(4, k)$ such that $n_{3}=n_{1}=3, n_{0}=0, c=0$ and $n_{2}=n-6$. As $G$ is connected there are three possibilities to construct such a graph, these possibilities are exposed in the subsections 3.3.1, 3.3.2, 3.3.3. 


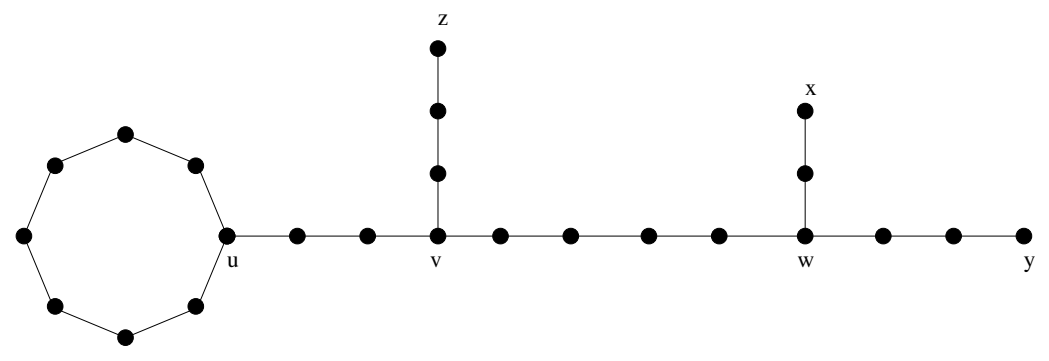

Figure 7: A graph $G \in \mathcal{G}_{1}$

\subsubsection{Unicyclic graphs with exactly three vertices of maximal degree 3 of which only one belongs to the cycle}

Let $T$ be a tree with exactly two vertices of maximal degree 3 . Let $\mathcal{G}_{1}$ be the set of the coalescences of $\mathrm{T}$ with a pendant vertex as distinguished vertex and a cycle $C_{p}, p \geq 6$. In the following we assume that the vertex of degree 3 belonging to the cycle is denoted by $u$ and $v, w$ are the other two vertices of degree 3 such that $v$ is between $u$ and $w ; x, y, z$ are the pendant vertices of $G$ such that $d(z, v)<d(z, w)$ and $d(x, w) \leq d(y, w)$. An example is given in figure 7 .

The aim of this section is to show the following theorem whose proof is summed up in table 1:

Theorem 19. The lollipop $L(4, k)$ cannot be cospectral with a graph $G \in \mathcal{G}_{1}$.

As $L(4, k)$ cannot be cospectral with a non-bipartite graph we suppose in the following that a graph $G \in \mathcal{G}_{1}$ is bipartite (the length of the cycle is even).

Proposition 7. Let $G \in \mathcal{G}_{1}$. If one of the following properties is true:

i) $d(u, v)>2$

ii) $d(u, v)=2, d(v, w)>1$ and $d(y, w)>2$

iii) $d(u, v)=2, d(v, w) \geq 4, d(y, w) \geq 2$

then $G$ is not cospectral with a lollipop.

Proof. Let $p$ be the length of the cycle of $G$. If one of these properties is true then $G$ possesses an induced subgraph with twice the eigenvalue 2. By the interlacing theorem it cannot be cospectral with a lollipop (theorem 11).

This subgraph is $C_{p} \cup T_{r}$ (for an $r \in \mathbb{N}$ ) in the case $i$ ), $C_{p} \cup S_{1,3,3}$ in $i i$ ) and $C_{p} \cup S_{1,2,5}$ in $i$ iii).

Proposition 8. Let $G \in \mathcal{G}_{1}$. If one of the following properties is true:

i) $d(u, v)=1, d(v, w)=1$,

ii) $d(u, v)=1$ and $d(v, w)>1$ and $(d(v, z)>1$ or $d(x, w)>1$ or $d(y, w)>1)$,

iii) $d(u, v)>1$ and $d(v, w)>1$ and $((d(v, z)>1$ and $d(y, w)>1)$ or $d(x, w)>1)$, 


\begin{tabular}{|c|c|c|c|c|c|c|c|}
\hline \multicolumn{6}{|c|}{ Graph } & Tool & Prop. \\
\hline \multicolumn{6}{|c|}{$\overline{p p=6}$} & $\overline{\mid \sum \lambda_{i}^{6}}$ & $8 v)$ \\
\hline \multirow{14}{*}{$p \geq 8$} & \multirow{3}{*}{$\begin{array}{c}d(u, v) \\
\quad=1\end{array}$} & \multicolumn{4}{|c|}{$d(v, w)=1$} & $\sum \lambda_{i}^{6}$ & $8 i)$ \\
\hline & & \multirow{2}{*}{$\begin{array}{c}d(v, w) \\
\quad>1\end{array}$} & \multicolumn{3}{|c|}{$\begin{array}{c}d(v, z)>1 \text { or } d(x, w)>1 \\
\text { or } d(y, w)>1\end{array}$} & $\sum \lambda_{i}^{6}$ & $8 i i)$ \\
\hline & & & \multicolumn{3}{|c|}{$\begin{array}{c}d(v, z)=1 \text { and } d(x, w)=1 \\
\text { and } d(y, w)=1\end{array}$} & $Q_{G}(1)$ & 9 \\
\hline & \multirow{10}{*}{$\begin{array}{l}d(u, v) \\
\quad=2\end{array}$} & \multirow{8}{*}{$\begin{array}{l}d(x, w) \\
\quad=1\end{array}$} & \multicolumn{3}{|c|}{$d(y, w)=1$} & $Q_{G}(2)$ & 11 \\
\hline & & & \multirow{5}{*}{$\begin{array}{c}d(y, w) \\
\quad=2\end{array}$} & \multirow{2}{*}{$d(v, z)>1$} & $d(v, w)=1$ & $\sum \lambda_{i}^{6}$ & $8 i v)$ \\
\hline & & & & & $d(v, w)>1$ & $\sum \lambda_{i}^{6}$ & $8 \mathrm{iii)}$ \\
\hline & & & & \multirow{3}{*}{$d(v, z)=1$} & $d(v, w)=1$ & $\sum \lambda_{i}^{6}$ & $8 i v)$ \\
\hline & & & & & $2 \leq d(v, w) \leq 3$ & $\sum \lambda_{i}^{8}$ & 10 \\
\hline & & & & & $d(v, w) \geq 4$ & $\overline{\lambda_{2} \geq 2}$ & $7 \mathrm{iii)}$ \\
\hline & & & \multirow{2}{*}{$\begin{array}{l}d(y, w) \\
>2\end{array}$} & \multicolumn{2}{|c|}{$d(v, w)=1$} & $\sum \lambda_{i}^{6}$ & $8 i v)$ \\
\hline & & & & \multicolumn{2}{|c|}{$d(v, w)>1$} & $\lambda_{2} \geq 2$ & $7 \mathrm{ii)}$ \\
\hline & & $d(x, w)$ & \multicolumn{3}{|c|}{$d(v, w)=1$} & $\sum \lambda_{i}^{6}$ & $8 i v)$ \\
\hline & & $>1$ & \multirow{2}{*}{\multicolumn{3}{|c|}{$\begin{array}{r}d(v, w)>1 \\
d(u, v)>2\end{array}$}} & $\sum \lambda_{i}^{6}$ & $8 \mathrm{iii})$ \\
\hline & \multicolumn{3}{|c|}{$d(u, v)>2$} & & & $\lambda_{2} \geq 2$ & $7 i)$ \\
\hline
\end{tabular}

Table 1: Proof of theorem 19 using a case disjunction over the possibilities for the values of $d$.

iv) $d(v, w)=1$ and $(d(v, z)>1$ or $d(y, w)>1$ or $d(x, w)>1)$,

v) $p=6$.

then

$$
\sum_{\lambda_{i} \in S p(G)} \lambda_{i}^{6}>20 n+96
$$

and $G$ cannot be cospectral with $L(4, k)$.

Proof. For the cases from $i)$ to $i v)$ we have $\left|P_{2}(G)\right|=n,\left|P_{3}(G)\right|=n+3,\left|S_{1,1,1}(G)\right|=$ $3,\left|P_{4}(G)\right|>n+4$ and apply proposition 1 .

For the case $v)$ we have $\left|P_{2}(G)\right|=n,\left|P_{3}(G)\right|=n+3,\left|S_{1,1,1}(G)\right|=3,\left|P_{4}(G)\right|>$ $n+2,\left|C_{6}(G)\right|=1$ and apply proposition 1 .

Proposition 9. Let $G \in \mathcal{G}_{1}$ such that $d(u, v)=1$ and $d(w, x)=d(w, y)=d(v, z)=1$. Then $G$ cannot be cospectral with $L(4, k)$.

Proof. Let $G \in \mathcal{G}_{1}$, with $n=p+q$ vertices where $p$ is the length of the cycle. We have:

$$
\begin{aligned}
Q_{G}(X)= & Q_{C_{p}}(X) Q_{S_{1,1, q-3}}(X)-X Q_{P_{p-1}}(X) Q_{S_{1,1, q-5}}(X) \\
= & X Q_{C_{p}}(X)\left(Q_{P_{q-1}}(X)-Q_{P_{q-3}}(X)\right) \\
& -X^{2} Q_{P_{p-1}}(X)\left(Q_{P_{q-3}}(X)-Q_{P_{q-5}}(X)\right)
\end{aligned}
$$

Using property 3 we compute $Q_{G}(1)$, the result depends on $\bar{p}$ and $\bar{q}$ which are $p$ and $q$ modulo 6 and are summed up into the following table: 


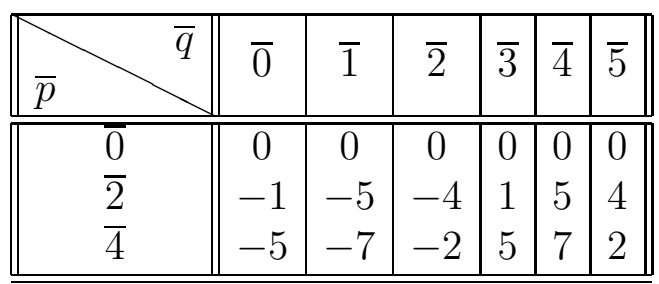

Comparing these results with proposition $4(\bar{n}=\bar{p}+\bar{q})$ we conclude that $G$ cannot be cospectral with $L(4, k)$.

Proposition 10. Let $G \in \mathcal{G}_{1}$ such that $p \geq 8, d(u, v)=2,2 \leq d(v, w) \leq 3, d(y, w)=2$, $d(v, z)=1, d(x, w)=1$. Then $G$ cannot be cospectral with $L(4, k)$.

Proof. We have $\left|P_{2}(G)\right|=n,\left|P_{3}(G)\right|=n+3,\left|P_{4}(G)\right|=n+4,\left|S_{1,1,2}(G)\right|=7$, $\left|P_{5}(G)\right|=n+6$ if $d(v, w)=2$ and $\left|P_{5}(G)\right|=n+5$ if $d(v, w) \geq 3$ and by proposition 1 :

$$
\sum \lambda_{i}^{8}= \begin{cases}70 n+588+16\left|C_{8}(G)\right| & \text { if } d(v, w)=2 \\ 70 n+580+16\left|C_{8}(G)\right| & \text { if } d(v, w)=3\end{cases}
$$

- If $d(v, w)=2$ then, by proposition $3, G$ cannot be cospectral with $L(4, k)$.

- If $d(v, w)=3$ then, by proposition $3, G$ is cospectral with $L(4, k)$ only if $p=8$. We then check that such a graph $G$ (drawn on figure 8) is not cospectral with $L(4,13)$ by comparing spectral radii (see tables 11 and 12 in appendix).

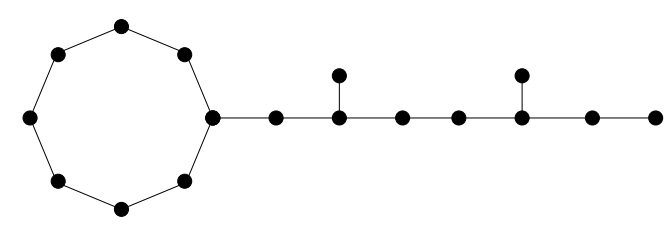

Figure 8:

Proposition 11. Let $G \in \mathcal{G}_{1}$ such that $d(u, v)=2, d(x, w)=d(y, w)=1$. Then $Q_{G}(2)=-4 p$ and $G$ cannot be cospectral with a lollipop $L(4, k)$

Proof. Set $b=d(v, w)$ and $a=d(z, v)$, using theorems 4 and 6 we have

$$
Q_{G}(X)=Q_{C_{p}}(X) Q_{T}(X)-Q_{P_{p-1}}(X) Q_{S_{1,1, a+b-1}}(X)
$$

(where $T$ is a tree) and using property 2 we get $Q_{G}(2)=0-p \times 4=-4 n+4(n-p)$. As $n-p>4$, proposition 5 implies that $G$ cannot be cospectral with a lollipop $L(4, k)$. 


\subsubsection{Unicyclic graphs with exactly three vertices of maximum degree 3 of which exactly two belong to the cycle}

Let $T$ be a tree with exactly one vertex $w$ of maximum degree 3 and $L(p, k), p \geq 6$, a lollipop (the vertex of degree 3 is denoted by $v$ and the pendant vertex by $z$ ). Let $\mathcal{G}_{2}$ be the set of coalescences of a lollipop with a vertex $u$ of degree 2 of the cycle as distinguished vertex and $T$ with a pendant vertex as distinguished vertex. The pendant vertices different from $z$ are denoted by $x$ and $y$ such that $d(x, w) \leq d(y, w)$. Such a graph is drawn in figure 9 .

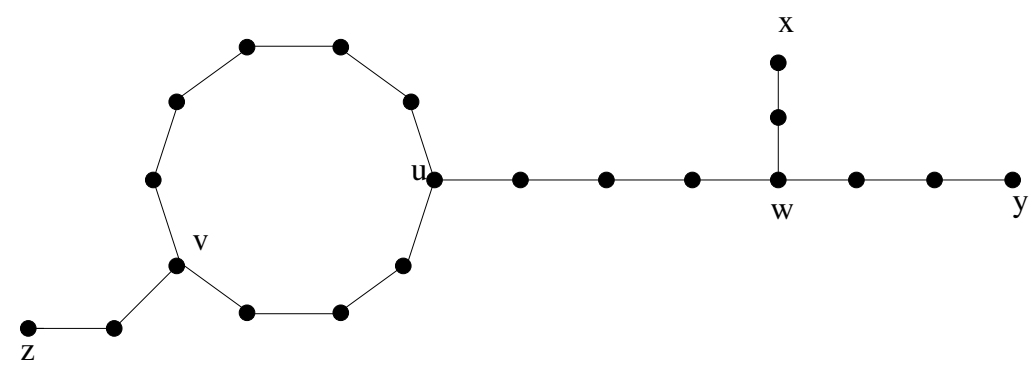

Figure 9: A graph $G \in \mathcal{G}_{2}$

The aim of this section is to show the following theorem whose proof is summed up in table 2 .

Theorem 20. A $L(4, k)$ cannot be cospectral with a graph $G \in \mathcal{G}_{2}$.

As in the previous section we can assume the length of the cycle of $G$ is even.

Proposition 12. Let $G \in \mathcal{G}_{2}$. If one of the following properties is true

i) $d(x, w)>1$ or $(d(y, w)>1$ and $d(z, v)>1)$,

ii) $d(u, v)=1$ and $(d(z, v)>1$ or $d(y, w)>1)$,

iii) $d(u, v)=1$ and $d(u, w)=1$,

iv) $d(u, w)=1$ and $(d(z, v)>1$ or $d(y, w)>1)$,

v) $p=6$,

then

$$
\sum_{i} \lambda_{i}^{6}>20 n+96
$$

and $G$ cannot be cospectral with a lollipop $L(4, k)$.

Proof. For all cases we have $\left|P_{2}(G)\right|=n,\left|P_{3}(G)\right|=n+3,\left|S_{1,1,1}(G)\right|=3$. Moreover, for the cases i) to iv) $\left|P_{4}(G)\right|>n+4$ and for the case v) $\left|P_{4}(G)\right|>n+2$ and $\left|C_{6}(G)\right|=1$ and we apply proposition 1 .

Proposition 13. Let $G \in \mathcal{G}_{2}$ such that $p \geq 8, d(u, v)>1, d(u, w)>1, d(z, v)=$ $1, d(w, x)=d(w, y)=1$, then $\sum_{i} \lambda_{i}^{6}<20 n+96$ and $G$ cannot be cospectral with a lollipop $L(4, k)$. 


\begin{tabular}{|c|c|c|c|c|c|c|}
\hline \multicolumn{5}{|c|}{ Graph } & Tool & Prop. \\
\hline \multicolumn{5}{|c|}{$p=6$} & $\sum \lambda_{i}^{6}$ & $12 v)$ \\
\hline \multirow{3}{*}{\multicolumn{2}{|c|}{$d(u, v)=1$}} & \multicolumn{3}{|c|}{$d(v, z)>1$ or $d(x, w)>1$ or $d(y, w)>1$} & $\sum \lambda_{i}^{6}$ & $12 \mathrm{ii})$ \\
\hline & & \multicolumn{3}{|c|}{$d(w, u)=1$} & $\sum \lambda_{i}^{6}$ & $12 \mathrm{iii})$ \\
\hline & & \multicolumn{3}{|c|}{$\begin{array}{c}d(v, z)=1 \text { and } \\
d(x, w)=1 \text { and } \\
d(y, w)=1 \text { and } d(w, u)>1\end{array}$} & $\begin{array}{l}Q_{G}(2) \\
\text { and } \\
Q_{G}(1)\end{array}$ & 14 \\
\hline \multirow{6}{*}{$p \geq 8$} & \multirow{6}{*}{$d(u, v)>1$} & $d(x, w)>1$ & $(d(y, w)>$ & nd $d(z, v)>1)$ & $\sum \lambda_{i}^{6}$ & $12 i)$ \\
\hline & & \multirow{2}{*}{$\begin{array}{l}d(x, w)=1 \\
\text { and } \\
d(y, w)=1\end{array}$} & \multirow[t]{2}{*}{$d(u, w)=1$} & $\begin{array}{c}d(v, z)>1 \text { or } \\
d(y, w)>1\end{array}$ & $\sum \lambda_{i}^{6}$ & $12 i v)$ \\
\hline & & & & $\begin{array}{c}d(v, z)=1 \text { and } \\
d(y, w)=1\end{array}$ & $Q_{G}(2)$ & 15 \\
\hline & & \multirow{3}{*}{$\begin{array}{c}\text { or } \\
d(x, w)=1 \\
\text { and } \\
d(z, v)=1\end{array}$} & \multirow[t]{3}{*}{$d(u, w)>1$} & $\begin{array}{c}d(v, z)=1 \text { and } \\
d(y, w)=1\end{array}$ & $\sum \lambda_{i}^{6}$ & 13 \\
\hline & & & & $\begin{array}{c}d(v, z)=1 \text { and } \\
d(y, w)>1\end{array}$ & & 16 \\
\hline & & & & $\begin{array}{c}d(v, z)>1 \text { and } \\
d(y, w)=1\end{array}$ & & 17 \\
\hline
\end{tabular}

Table 2: Proof of theorem 20 using a case disjunction over the possibilities for the values of $d$. An empty cell in the column tool means that the proof uses more than three tools.

Proof. The subgraphs $M$ of $G$ with $w_{6}(G)>0$ are $P_{2}, P_{3}, P_{4}, S_{1,1,1}$ and $\left|P_{2}(G)\right|=n$, $\left|P_{3}(G)\right|=n+3,\left|S_{1,1,1}(G)\right|=3,\left|P_{4}(G)\right|=n+3$ and we apply proposition 1 .

Proposition 14. Let $G \in \mathcal{G}_{2}$ such that $d(u, v)=1, d(z, v)=1, d(w, y)=1, d(w, u)>1$, then $G$ is not cospectral with $L(4, k)$.

Proof. Since $d(w, x) \leq d(w, y)=1$ we have $d(w, x)=1$. Let $\alpha=d(u, w)$ (so $n=p+\alpha+3)$, by theorem 4 we get:

$$
\begin{aligned}
Q_{G}(X) & =Q_{L(p, 1)}(X) Q_{S_{1,1, \alpha-1}}(X)-Q_{P_{p}}(X) Q_{S_{1,1, \alpha-2}}(X) \\
& =\left(X Q_{C_{p}}(X)-Q_{P_{p-1}}(X)\right) Q_{S_{1,1, \alpha-1}}(X)-Q_{P_{p}}(X) Q_{S_{1,1, \alpha-2}}(X)
\end{aligned}
$$

and (with property 2) $Q_{G}(2)=-8 p-4$. So $Q_{G}(2)=Q_{L(4, k)}(2)$ if and only if $-8 p-4=$ $-4 n+16$ that is $\alpha=p+2$.

As a consequence

$$
\begin{aligned}
Q_{G}(X)= & \left(X Q_{C_{p}}(X)-Q_{P_{p-1}}(X)\right) Q_{S_{1,1, p+1}}(X)-Q_{P_{p}}(X) Q_{S_{1,1, p}}(X) \\
= & \left(X Q_{C_{p}}(X)-Q_{P_{p-1}}(X)\right) X\left(Q_{P_{p+3}}(X)-Q_{P_{p+1}}(X)\right) \\
& -Q_{P_{p}}(X) X\left(Q_{P_{p+2}}(X)-Q_{P_{p}}(X)\right)
\end{aligned}
$$

By property 3 we have (let's note that $n=2 p+5$ ):

- If $\bar{p}=\overline{0}($ so $\bar{n}=\overline{5})$ then $Q_{G}(1)=1$. 
- If $\bar{p}=\overline{2}($ so $\bar{n}=\overline{3})$ then $Q_{G}(1)=-4$.

- If $\bar{p}=\overline{4}($ so $\bar{n}=\overline{1})$ then $Q_{G}(1)=0$.

where $\bar{p}$ and $\bar{n}$ are $p$ and $n$ modulo 6 . And by proposition $4, G$ is not cospectral with $L(4, k)$.

Proposition 15. Let $G \in \mathcal{G}_{2}$ such that $d(u, v)>1, d(w, x)=d(w, y)=d(v, z)=$ $d(u, w)=1$, then $G$ cannot be cospectral with $L(4, k)$.

Proof. Set $a=d(u, v)$ and $b=p-a$. We have:

$$
\begin{aligned}
Q_{G}(X) & =Q_{L(p, 1)}(X) Q_{P_{3}}(X)-X^{2} Q_{S_{1, a-1, b-1}}(X) \\
& =\left(X Q_{C_{p}}(X)-Q_{P_{p-1}}(X)\right) Q_{P_{3}}(X)-X^{2} Q_{S_{1, a-1, b-1}}(X)
\end{aligned}
$$

and $Q_{G}(2)=-4 p-4(2 a+2 b-a b)$. As $n=p+4$ we have $Q_{G}(2)+4 n-16=-4(2 p-a b)$ so $Q_{G}(2)+4 n-16=0$ if and only if $a b=2 p$.

- If $a=2$ then $2 b=4+2 b$, impossible.

- If $a=3$ then $b=6$ and $p=9, p$ odd is impossible.

- If $a=4$ then $b=4$ and $p=8$ we check that this graph is not cospectral with $L(4,8)$.

- If $a>4$ then as $p \leq 2 b$ we have $2 p-a b<0$.

As a result $G$ is not cospectral with $L(4, k)$.

Proposition 16. Let $G \in \mathcal{G}_{2}$ such that $p \geq 8, d(u, v)>1, d(w, u)>1, d(w, y)>1$, $d(w, x)=d(v, z)=1$. Then $G$ is not cospectral with a lollipop $L(4, k)$.

Proof. Let $a=d(u, v), b=p-a, \alpha=d(u, w), \beta=d(w, y) \geq 2$. We have $a \leq b$ and $p \leq 2 b$ and $n=p+\alpha+\beta+2$.

$$
\begin{aligned}
Q_{G}(X) & =Q_{L(p, 1)}(X) Q_{S_{1, \alpha-1, \beta}}(X)-Q_{S_{1, a-1, b-1}}(X) Q_{S_{1, \alpha-2, \beta}}(X) \\
& =\left(X Q_{C_{p}}(X)-Q_{P_{p-1}}(X)\right) Q_{S_{1, \alpha-1, \beta}}(X)-Q_{S_{1, a-1, b-1}}(X) Q_{S_{1, \alpha-2, \beta}}(X)
\end{aligned}
$$

Using property 2 we obtain

$$
Q_{G}(2)=-p(\alpha+2 \beta-\alpha \beta+2)-(2 a+2 b-a b)(\alpha+3 \beta-\alpha \beta+1)
$$

The following inequality will be useful: $a b=(a-1)(b-1)+p-1 \geq b-1+p-1 \geq \frac{3}{2} p-2$. The main argument of this proof is that $Q_{G}(2) \neq-4 n+16$ so $G$ cannot be cospectral with a lollipop $L(4, k)$ (proposition 5$)$. 
- Case $\beta=2$. $Q_{G}(2)-(-4 n+16)=(3 \alpha-16) p+(7-\alpha) a b+4 \alpha$.

- If $\alpha=2$ then $Q_{G}(2)-(-4 n+16)=-10 p+5 a b+8 \neq 0$ (otherwise 5 divides 8)

- If $\alpha=3$ then $Q_{G}(2)-(-4 n+16)=-7 p+4 a b+12$. If $a \geq 4$ then $-7 p+4 a b+12>$ 0 (because $p \leq 2 b$ ). If $a=3$ then $-7 p+4 a b+12=5 b-9 \neq 0$ (because $b \in \mathbb{N}$ ). If $a=2$ then $-7 p+4 a b+12=b-2 \neq 0$ (because $a+b=p \geq 8$ ).

- If $4 \leq \alpha \leq 7$ then

$$
\begin{aligned}
Q_{G}(2)-(-4 n+16) & \geq(3 \alpha-16) p+(7-\alpha)\left(\frac{3}{2} p-2\right)+4 \alpha \\
& \geq\left(\frac{3}{2} \alpha-\frac{11}{2}\right) p-14+6 \alpha \\
& \geq \frac{p}{2}+10>0
\end{aligned}
$$

- If $\alpha>7$ then the disjoint union $C_{p} \cup S_{1,2,5}$ is an induced subgraph of $G$ with twice the eigenvalue 2 and by the interlacing theorem and theorem 11, $G$ is not cospectral with a lollipop.

- Case $\beta \geq 3$ :

- $\alpha=2$. We have $\left|P_{2}(G)\right|=n,\left|P_{3}(G)\right|=n+3,\left|P_{4}(G)\right|=n+4,\left|S_{1,1,1}(G)\right|=3$, $\left|S_{1,1,2}(G)\right|=7,\left|P_{5}(G)\right|=n+6$ if $a>2$ and $\left|P_{5}(G)\right|=n+7$ if $a=2$. By proposition 1 we have $\sum \lambda_{i}^{8}=70 n+588+16\left|C_{8}(G)\right|$ if $a>2$ and in that case $G$ in not cospectral with $L(4, k)$ (proposition 3). If $a=2$ then $Q_{G}(2)=-4 p-4(\beta+3)=-4 n+4 \neq-4 n+16$.

$-\alpha=3 . Q_{G}(2)+4 n-16=-p(-\beta+5)-(2 p-a b) \times 4+4(p+\beta+5)-16=p(\beta-$ $9)+4 a b+4 \beta+4$. But $\beta \geq 3$ and $a b \geq \frac{3}{2} p-2$, so $Q_{G}(2)+4 n-16 \geq 4 \beta-4>0$.

$-\alpha=4$.

* If $\beta \geq 5$ the disjoint union $C_{p} \cup S_{1,2,5}$ is an induced subgraph of $G$ with twice the eigenvalue 2 and by the interlacing theorem and theorem 11, $G$ is not cospectral with a lollipop.

$*$ If $\beta=4$ then $Q_{G}(2)=a b>0$ and $Q_{L(4, k)}(2)<0$

$*$ If $\beta=3$ then $Q_{G}(2)=2(a b-2 p), n=p+9$ and $Q_{G}(2)-(-4 n+16)=$ $2 a b+20>0$

- $\alpha>4$. The disjoint union $C_{p} \cup S_{1,3,3}$ is an induced subgraph of $G$ with twice the eigenvalue 2 and by the interlacing theorem and theorem 11, $G$ is not cospectral with a lollipop. 
Property 5. Let $r \in \mathbb{R}, r>2$, we have $Q_{P_{n}}(r)=\alpha_{1} \beta_{1}^{n}+\alpha_{2} \beta_{2}^{n}$ with $\beta_{1}=\frac{r+\sqrt{r^{2}-4}}{2}>1$, $\beta_{2}=\frac{r-\sqrt{r^{2}-4}}{2}<1, \alpha_{1}=\frac{r-\beta_{2}}{\beta_{1}-\beta_{2}}>1, \alpha_{2}=1-\alpha_{1}<0$.

Proof. Let $\left(u_{n}\right)_{n \in \mathbb{N}}$ be the sequence $u_{n}=Q_{P_{n}}(r)$. We have $u_{n}=r u_{n-1}-u_{n-2}$, so $u_{n}=\alpha_{1} \beta_{1}^{n}+\alpha_{2} \beta_{2}^{n}$ where $\beta_{1}, \beta_{2}$ are roots of $X^{2}-r X+1$ and we note that $1=u_{0}=\alpha_{1}+\alpha_{2}$, $r=u_{1}=\alpha_{1} \beta_{1}+\alpha_{2} \beta_{2}$.

Lemma 1. Let $G \in \mathcal{G}_{2}$ with $d(u, v)=2, d(w, x)=d(w, y)=1, d(v, z)>1, d(u, w)>$ $d(v, z)$, then $G$ is not cospectral with a $L(4, k)$.

Proof. Let $\alpha=d(u, w), l=d(v, z)$, we have $n=p+\alpha+l+2$. Applying theorem 5 to the vertex at distance 1 of $u$ and $v$, we have:

$$
\begin{aligned}
Q_{G}(X)= & X Q_{S_{1,1, n-4}}(X)-Q_{P_{l}}(X) Q_{S_{1,1, \alpha+p-3}}(X) \\
& -Q_{P_{l+p-2}}(X) Q_{S_{1,1, \alpha-1}}(X)-2 Q_{P_{l}}(X) Q_{S_{1,1, \alpha-1}}(X)
\end{aligned}
$$

and applying theorem 5 to the vertex of degree 2 of the cycle of $L(4, k)$ at distance 2 from the vertex of degree 3 , we have:

$$
Q_{L(4, k)}(X)=X Q_{S_{1,1, n-4}}(X)-2 Q_{P_{n-2}}(X)-2 Q_{P_{n-4}}(X)
$$

Noting that $Q_{S_{1,1, c}}=X\left(Q_{P_{c+2}}(X)-Q_{P_{c}}(X)\right)$ and $Q_{P_{n-2}}(X)+Q_{P_{n-4}}(X)=X Q_{P_{n-3}}(X)$ we have:

$$
\begin{aligned}
Q_{G}(X)-Q_{L(4, k)}(X)= & -X Q_{P_{l}}(X) Q_{P_{\alpha+p-1}}(X)+X Q_{P_{l}}(X) Q_{P_{\alpha+p-3}}(X) \\
& -X Q_{P_{l+p-2}}(X) Q_{P_{\alpha+1}}(X)+X Q_{P_{l+p-2}}(X) Q_{P_{\alpha-1}}(X) \\
& -2 X Q_{P_{l}}(X) Q_{P_{\alpha+1}}(X)+2 X Q_{P_{l}}(X) Q_{P_{\alpha-1}}(X) \\
& +2 X Q_{P_{n-3}}(X)
\end{aligned}
$$

According to the previous property, we have for $r>2$ :

$$
\begin{aligned}
Q_{G}(r)-Q_{L(4, k)}(r)= & -r \alpha_{1}^{2} \beta_{1}^{n-3}-r \alpha_{2}^{2} \beta_{2}^{n-3}-r \alpha_{1} \alpha_{2} \beta_{1}^{l} \beta_{2}^{\alpha+p-1}-r \alpha_{1} \alpha_{2} \beta_{1}^{\alpha+p-1} \beta_{2}^{l} \\
& +r \alpha_{1}^{2} \beta_{1}^{n-5}+r \alpha_{2}^{2} \beta_{2}^{n-5}+r \alpha_{1} \alpha_{2} \beta_{1}^{l} \beta_{2}^{\alpha+p-3}+r \alpha_{1} \alpha_{2} \beta_{1}^{\alpha+p-3} \beta_{2}^{l} \\
& -r \alpha_{1}^{2} \beta_{1}^{n-3}-r \alpha_{2}^{2} \beta_{2}^{n-3}-r \alpha_{1} \alpha_{2} \beta_{1}^{l+p-2} \beta_{2}^{\alpha+1}-r \alpha_{1} \alpha_{2} \beta_{1}^{\alpha+1} \beta_{2}^{l+p-2} \\
& +r \alpha_{1}^{2} \beta^{n-5}+r \alpha_{2}^{2} \beta^{n-5}+r \alpha_{1} \alpha_{2} \beta_{1}^{l+p-2} \beta_{2}^{\alpha-1}+r \alpha_{1} \alpha_{2} \beta_{1}^{\alpha-1} \beta_{2}^{l+p-2} \\
& -2 r \alpha_{1}^{2} \beta_{1}^{l+\alpha+1}-2 r \alpha_{2}^{2} \beta_{2}^{l+\alpha+1}-2 r \alpha_{1} \alpha_{2} \beta_{1}^{l} \beta_{2}^{\alpha+1}-2 r \alpha_{1} \alpha_{2} \beta_{1}^{\alpha+1} \beta_{2}^{l} \\
& +2 r \alpha_{1}^{2} \beta_{1}^{l+\alpha-1}+2 r \alpha_{2}^{2} \beta_{2}^{l+\alpha-1}+2 r \alpha_{1} \alpha_{2} \beta_{1}^{l} \beta_{2}^{\alpha-1}+2 r \alpha_{1} \alpha_{2} \beta_{1}^{\alpha-1} \beta_{2}^{l} \\
& +2 r \alpha_{1} \beta_{1}^{n-3}+2 r \alpha_{2} \beta_{2}^{n-3}
\end{aligned}
$$

Let $x=\alpha+p-l-1$ and $y=l+p-\alpha-1, z=\alpha+l+1, t=\alpha+1-l$, we have $x>y$, $x>-y+2, x>t, z>t$. Using $\beta_{2}=\beta_{1}^{-1}$, we have:

$$
\begin{aligned}
Q_{G}(r)-Q_{L(4, k)}(r)= & 2 r\left(\left(\alpha_{1}-\alpha_{1}^{2}\right) \beta_{1}^{2}+\alpha_{1}^{2}\right) \beta_{1}^{n-5}+2 r\left(\left(\alpha_{2}-\alpha_{2}^{2}\right) \beta_{2}^{2}+\alpha_{2}^{2}\right) \beta_{2}^{n-5} \\
& -2 r \alpha_{1}^{2} \beta_{1}^{z}-2 r \alpha_{2}^{2} \beta_{1}^{-z}+2 r \alpha_{1}^{2} \beta_{1}^{z-2}+2 r \alpha_{2}^{2} \beta_{1}^{-z+2} \\
& -r \alpha_{1} \alpha_{2}\left(\beta_{1}^{-x}+\beta_{1}^{x}-\beta_{1}^{x-2}-\beta_{1}^{-x+2}+\beta_{1}^{-y-2}+\beta_{1}^{-y+2}\right. \\
& \left.-\beta_{1}^{y}-\beta_{1}^{-y}+2 \beta_{1}^{-t}+2 \beta_{1}^{t}-2 \beta_{1}^{-t+2}-2 \beta_{1}^{t-2}\right)
\end{aligned}
$$


but we have the three following equalities:

$$
\begin{gathered}
\alpha_{1} \alpha_{2}=\alpha_{1}-\alpha_{1}^{2}=\frac{-1}{r^{2}-4} \\
\left(\alpha_{1}-\alpha_{1}^{2}\right) \beta_{1}^{2}+\alpha_{1}^{2}=0 \\
\left(\alpha_{2}-\alpha_{2}^{2}\right) \beta_{2}^{2}+\alpha_{2}^{2}=0
\end{gathered}
$$

so

$$
\begin{aligned}
Q_{G}(r)-Q_{L(4, k)}(r)= & -2 r \alpha_{1}^{2} \beta_{1}^{z}-2 r \alpha_{2}^{2} \beta_{1}^{-z}+2 r \alpha_{1}^{2} \beta_{1}^{z-2}+2 r \alpha_{2}^{2} \beta_{1}^{-z+2} \\
& +\frac{r}{r^{2}-4}\left(\beta_{1}^{x}+\beta_{1}^{-x}-\beta_{1}^{x-2}-\beta_{1}^{-x+2}+\beta_{1}^{-y-2}+\beta_{1}^{-y+2}\right. \\
& \left.-\beta_{1}^{y}-\beta_{1}^{-y}+2 \beta_{1}^{-t}+2 \beta_{1}^{t}-2 \beta_{1}^{-t+2}-2 \beta_{1}^{t-2}\right)
\end{aligned}
$$

and we have

$$
\lim _{r \rightarrow+\infty} Q_{G}(r)-Q_{L(4, k)}(r)=\left\{\begin{array}{l}
+\infty \text { if } z+1<x-1 \\
-\infty \text { if } z+1 \geq x-1
\end{array}\right.
$$

and $G$ is not cospectral with $L(4, k)$.

Proposition 17. Let $G \in \mathcal{G}_{2}$ with $p \geq 8, d(u, v)>1, d(w, x)=d(w, y)=1, d(v, z)>1$, $d(u, w)>1$, then $G$ is not cospectral with a $L(4, k)$.

Proof. We distinguish the following cases:

- case 1: $d(u, v)>2$ and $d(u, w)>2$ and $d(z, v)>2$

- case 2: $d(u, v)>2$ and $d(u, w)>2$ and $d(z, v)=2$

- case 3: $d(u, v)>2$ and $d(u, w)=2$ and $d(z, v)>2$

- case 4: $d(u, v)>2$ and $d(u, w)=2$ and $d(z, v)=2$

- case $5: d(u, v)=2$

- For cases 1 and 4 we have $\left|P_{2}(G)\right|=n,\left|P_{3}(G)\right|=n+3,\left|P_{4}(G)\right|=n+4,\left|S_{1,1,1}(G)\right|=$ 3, $\left|S_{1,1,2}(G)\right|=7,\left|P_{5}(G)\right|=n+6,|L(4,1)(G)|=0,|L(4,2)(G)|=0$ so (proposition 1) $\sum \lambda_{i}^{8}=70 n+588+16\left|C_{8}(G)\right|$ and $G$ is not cospectral with $L(4, k)$ (proposition 3).

- For cases 2, 3 and 5, let us compute $Q_{G}(2)$. Let $a=d(u, v), b=p-a, \alpha=d(u, w)$, $l=d(v, z)$.

$$
\begin{aligned}
Q_{G}(X)= & Q_{L(p, l)}(X) Q_{S_{1,1, \alpha-1}}(X)-Q_{S_{a-1, b-1, l}}(X) Q_{S_{1,1, \alpha-2}}(X) \\
= & \left(P_{C_{p}}(X) Q_{P_{l}}(X)-Q_{P_{p-1}}(X) Q_{P_{l-1}}(X)\right) Q_{S_{1,1, \alpha-1}}(X) \\
& -Q_{S_{a-1, b-1, l}}(X) Q_{S_{1,1, \alpha-2}}(X)
\end{aligned}
$$

Using property 2 we have $Q_{G}(2)+4 n-16=-8 l p+4 a b l+4 \alpha+4 l-8$ and $G$ is cospectral with $L(4, k)$ only if $Q_{G}(2)+4 n-16=0$ that is $\alpha=l(2 p-a b-1)+2$. 
- For case 3 we have $\alpha=2$ so $2 p-a b-1=0$ and $a$ is odd. If $a=3$ then $b=5$ and $p=8$. We have $\left|P_{2}(G)\right|=n,\left|P_{3}(G)\right|=n+3,\left|P_{4}(G)\right|=n+4$, $\left|P_{5}(G)\right|=n+7,\left|S_{1,1,1}(G)\right|=3,\left|S_{1,1,2}(G)\right|=7,\left|C_{8}(G)\right|=1$. So $\sum \lambda_{i}^{8}=70 n+612$ and in this case $G$ is not cospectral with $L(4, k)$ (proposition 3). If $a \geq 5$ then $2 p-a b-1 \leq 4 b-5 b-1<0$ and this finishes the case 3 .

- For case $2,\left|P_{2}(G)\right|=n,\left|P_{3}(G)\right|=n+3,\left|P_{4}(G)\right|=n+4,\left|S_{1,1,1}(G)\right|=3,\left|S_{1,1,2}(G)\right|=$ $7,\left|P_{5}(G)\right|=n+5,|L(4,1)(G)|=0,|L(4,2)(G)|=0$ so (proposition 1) $\sum \lambda_{i}^{8}=$ $70 n+580+16\left|C_{8}(G)\right|$ and $G$ is cospectral with $L(4, k)$ only if $p=8$. We have $l=2$ and $\alpha=l(2 p-a b-1)+2$ so the graphs that can be cospectral with $L(4, k)$ are the ones with $a=3, b=5$ so $\alpha=2$, impossible, or $a=4, b=4$ so $\alpha=0$, impossible.

- For case $5, G$ is cospectral with $L(4, k)$ only if $\alpha=3 l+2$, but this is impossible according to lemma 1.

\subsubsection{Unicyclic graphs with exactly three vertices of maximum degree 3 , all of them belonging to the cycle}

Let $\mathcal{G}_{3}$ be the set of the graphs $G$ obtained in the following way:

- Coalesce a lollipop $L(p, k), p \geq 6, k \geq 1$ with a vertex of degree 2 of the cycle as distinguished vertex and a path with a pendant vertex as distinguished vertex.

- Coalesce the previous graph with a vertex of the cycle of degree 2 as distinguished vertex and a path with a pendant vertex as distinguished vertex.

We denote by $u_{1}, u_{2}, u_{3}$ the three vertices of degree 3 and by $x_{1}, x_{2}, x_{3}$ the pendant vertices such that $d\left(x_{i}, u_{i}\right)=\min _{j} d\left(x_{i}, u_{j}\right)$. An example is given in figure 10 .

The aim of this section is to show the following theorem whose proof is summed up in table 3:

Theorem 21. A lollipop $L(4, k)$ cannot be cospectral with a graph $G \in \mathcal{G}_{3}$.

As in the previous sections we assume that the cycle of $G$ is even.

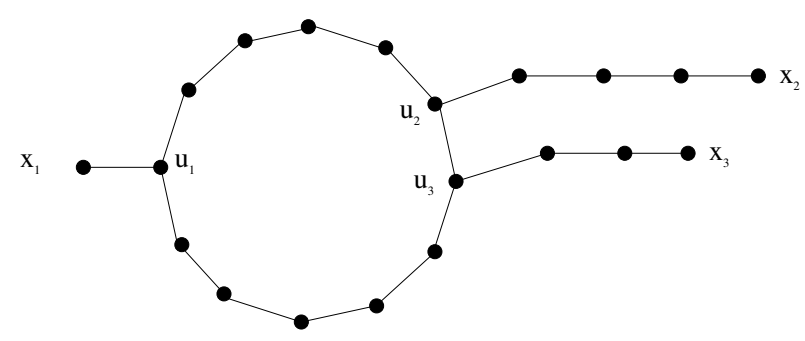

Figure 10: A graph $G \in \mathcal{G}_{3}$ 


\begin{tabular}{|c|c|c|c|c|c|c|c|}
\hline \multicolumn{6}{|c|}{ Graph } & Tool & $\begin{array}{l}\text { Propo- } \\
\text { sition }\end{array}$ \\
\hline \multicolumn{6}{|c|}{$p=6$} & $\sum \lambda_{i}^{6}$ & $18 i v)$ \\
\hline \multicolumn{6}{|c|}{$\exists i, j, i \neq j: d\left(x_{i}, u_{i}\right)>1$ and $d\left(x_{j}, u_{j}\right)>1$} & $\sum \lambda_{i}^{6}$ & $18 i)$ \\
\hline \multicolumn{6}{|c|}{$\exists r, s, t, r \neq s, s \neq t, r \neq t,: d\left(u_{r}, u_{s}\right)=1$ and $d\left(u_{s}, u_{t}\right)=1$} & $\sum \lambda_{i}^{6}$ & $18 i i)$ \\
\hline & \multirow{3}{*}{$d\left(x_{k}, u_{k}\right)=1$} & \multicolumn{4}{|c|}{$d\left(u_{r}, u_{t}\right)>1$} & $\sum \lambda_{i}^{6}$ & 19 \\
\hline \multirow{3}{*}{$\begin{array}{c}p \geq 8 \\
\text { and }\end{array}$} & & \multirow{2}{*}{$d\left(u_{r}, u_{t}\right)=1$} & \multirow{2}{*}{\multicolumn{3}{|c|}{$d\left(u_{r}, u_{s}\right)=2$ or $d\left(u_{s}, u_{t}\right)=2$}} & $\sum \lambda_{i}^{8}$ & 20 \\
\hline & & & $d\left(u_{r}, u_{s}\right)$ & & & $\sum \lambda_{i}^{8}$ & 20 \\
\hline & \multirow{7}{*}{$d\left(x_{k}, u_{k}\right)>1$} & \multicolumn{4}{|c|}{$d\left(u_{r}, u_{t}\right)=1$} & $\sum \lambda_{i}^{6}$ & 18 iii) \\
\hline \multirow{6}{*}{$\begin{array}{c}\exists l, \jmath, k \\
\text { two by two } \\
\text { distinct } \\
\exists r, s, t \\
\text { two by two } \\
\text { distinct: } \\
d\left(x_{i}, u_{i}\right)=1 \\
d\left(x_{j}, u_{j}\right)=1 \\
d\left(x_{k}, u_{k}\right) \geq 1 \\
d\left(u_{r}, u_{s}\right)>1 \\
d\left(u_{s}, u_{t}\right)>1\end{array}$} & & \multirow{6}{*}{$d\left(u_{r}, u_{t}\right)>1$} & \multicolumn{3}{|c|}{$d\left(x_{k}, u_{k}\right)=2$} & $\sum \lambda_{i}^{8}$ & 21 \\
\hline & & & \multicolumn{3}{|c|}{$\forall l_{1}, l_{2}, d\left(u_{l_{1}}, u_{l_{2}}\right)>2$} & $\sum \lambda_{i}^{8}$ & 22 iii) \\
\hline & & & \multicolumn{3}{|c|}{$\begin{array}{c}\exists r, s, t \\
d\left(u_{r}, u_{s}\right)=2 \text { and } \\
d\left(u_{r}, u_{t}\right)>2 \text { and } \\
d\left(u_{s}, u_{t}\right)>2\end{array}$} & $\sum \lambda_{i}^{8}$ & $22 i i)$ \\
\hline & & & \multirow{3}{*}{$d\left(x_{k}, u_{k}\right)>2$} & \multirow{2}{*}{$\begin{aligned} d\left(u_{i}, u_{j}\right) & =2 \\
\text { and } & \\
d\left(u_{j}, u_{k}\right) & =2\end{aligned}$} & $p=8$ & $\sum \lambda_{i}^{8}$ & $22 i)$ \\
\hline & & & & & $p \geq 10$ & $\sum \lambda_{i}^{10}$ & 23 \\
\hline & & & & \multicolumn{2}{|c|}{$\begin{aligned} d\left(u_{i}, u_{k}\right) & =2 \\
\text { and } & \\
d\left(u_{j}, u_{k}\right) & =2\end{aligned}$} & $\begin{array}{l}Q_{G}(2) \\
\text { and } \\
R(0) \\
\end{array}$ & 24 \\
\hline
\end{tabular}

Table 3: Proof of theorem 21 using a case disjunction over the possibilities for the values of $d$. $R$ denotes the polynomial $R(X)=\frac{Q_{G}(X)}{X^{2}}$ 
Proposition 18. Let $G \in \mathcal{G}_{3}$. If one of the following properties is true:

i) $\exists i, j, i \neq j: d\left(x_{i}, u_{i}\right)>1, d\left(x_{j}, u_{j}\right)>1$,

ii) $\exists r, s, t, r \neq s, r \neq t, s \neq t: d\left(u_{r}, u_{s}\right)=d\left(u_{s}, u_{t}\right)=1$,

iii) $\exists i, r, t: d\left(x_{i}, u_{i}\right)>1, d\left(u_{r}, u_{t}\right)=1$,

iv) $p=6$,

then

$$
\sum_{i} \lambda_{i}^{6}>20 n+96
$$

and $G$ cannot be cospectral with a lollipop $L(4, k)$.

Proof. For cases i) to iii) we have $\left|P_{2}(G)\right|=n,\left|P_{3}(G)\right|=n+3,\left|S_{1,1,1}(G)\right|=$ $3,\left|P_{4}(G)\right|>n+4$ and we apply proposition 1 .

For case iv) we have $\left|P_{2}(G)\right|=n,\left|P_{3}(G)\right|=n+3,\left|S_{1,1,1}(G)\right|=3,\left|P_{4}(G)\right|>n+$ $2,\left|C_{6}(G)\right|=1$ and we apply proposition 1 .

Proposition 19. Let $G \in \mathcal{G}_{3}$ such that $p>6, \forall i, r, s, d\left(u_{i}, x_{i}\right)=1, d\left(u_{r}, u_{s}\right)>1$. Then

$$
\sum_{i} \lambda_{i}^{6}=20 n+90
$$

and $G$ cannot be cospectral with a lollipop $L(4, k)$.

Proof. We have $\left|P_{2}(G)\right|=n,\left|P_{3}(G)\right|=n+3,\left|P_{4}(G)\right|=n+3,\left|S_{1,1,1}(G)\right|=3$ and no $p$-cycle for $p \leq 6$. We conclude with proposition 1 .

The following three propositions compute $\sum \lambda_{i}^{8}$ for some $G \in \mathcal{G}_{3}$, their proofs are based on counting motifs $M \in \mathcal{M}_{8},|M(G)|>0$ which is done in a summary table 4 . In the following $\left|C_{8}(G)\right|$ is denoted by $c_{8}$.

Proposition 20. Let $G \in \mathcal{G}_{3}$ such that $p \geq 8, \forall i, d\left(u_{i}, x_{i}\right)=1$, and $\exists r, s, t$ two by two distinct: $d\left(u_{r}, u_{t}\right)=1, d\left(u_{r}, u_{s}\right)>1, d\left(u_{s}, u_{t}\right)>1$. Then:

$$
\sum_{i} \lambda_{i}^{8}= \begin{cases}70 n+588+16 c_{8} & \text { if } d\left(u_{r}, u_{s}\right)=2 \text { or } d\left(u_{s}, u_{t}\right)=2 \\ 70 n+580+16 c_{8} & \text { otherwise }\end{cases}
$$

and $G$ cannot be cospectral with a $L(4, k)$.

Proof. Using table 4, we apply proposition 1 to compute $\sum_{i} \lambda_{i}^{8}$. The only case for which $\sum \lambda_{i}^{8}=70 n+596$ is when $\forall i, d\left(u_{i}, x_{i}\right)=1, \exists r, s, t$ two by two distinct : $d\left(u_{r}, u_{t}\right)=$ $1, d\left(u_{r}, u_{s}\right)>2, d\left(u_{s}, u_{t}\right)>2$ and $c_{8}=1$. This case is drawn in figure 11 and we check that it is not cospectral with $L(4,7)$ by comparing spectral radii (see tables 11 and 12 in appendix). 


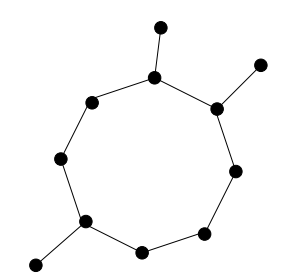

Figure 11:

Proposition 21. Let $G \in \mathcal{G}_{3}$ such that $p \geq 8, \exists i, j, k: d\left(u_{i}, x_{i}\right)=d\left(u_{j}, x_{j}\right)=$ $1, d\left(u_{k}, x_{k}\right)=2$. We distinguish the three following cases

- case 1: $\exists r, s, t, r \neq s, r \neq t, s \neq t: d\left(u_{r}, u_{s}\right)=d\left(u_{s}, u_{t}\right)=2$.

- case 2: $\exists r, s, t, r \neq s, r \neq t, s \neq t: d\left(u_{r}, u_{s}\right)=2$ and $d\left(u_{r}, u_{t}\right)>2$ and $d\left(u_{s}, u_{t}\right)>2$.

- case 3: $\forall s, t, d\left(u_{s}, u_{t}\right)>2$.

Then:

$$
\sum_{i} \lambda_{i}^{8}=\left\{\begin{array}{l}
70 n+588+16 c_{8} \text { for the case } 1 \\
70 n+580+16 c_{8} \text { for the case } 2 \\
70 n+572 \text { for the case } 3
\end{array}\right.
$$

and $G$ cannot be cospectral with a lollipop $L(4, k)$.

Proof. Using table 4, we apply proposition 1 to compute $\sum_{i} \lambda_{i}^{8}$. Under the hypotheses of the proposition, the only cases for which $\sum \lambda_{i}^{8}=70 n+596$ is when $c_{8}=1$ in case 2 . These cases are drawn in figure 12 and we check that they are not cospectral with $L(4,8)$ by comparing spectral radii (see tables 11 and 12 in appendix).
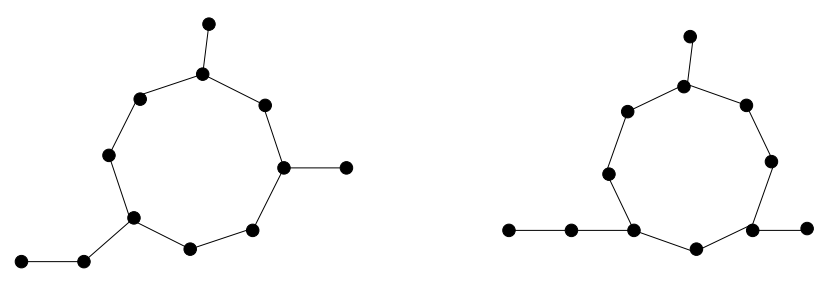

Figure 12:

Proposition 22. Let $G \in \mathcal{G}_{3}$ such that $p \geq 8, \exists i, j, k: d\left(u_{i}, x_{i}\right)=d\left(u_{j}, x_{j}\right)=$ $1, d\left(u_{k}, x_{k}\right)>2$. We distinguish the three following cases

- case 1: $\exists r, s, t, r \neq s, r \neq t, s \neq t: d\left(u_{r}, u_{s}\right)=d\left(u_{s}, u_{t}\right)=2$. 


\begin{tabular}{|c|c|c|c|c|}
\hline$M$ & $w_{8}(M)$ & $\left|M\left(G_{a}\right)\right|$ & $\left|M\left(G_{b}\right)\right|$ & $\left|M\left(G_{c}\right)\right|$ \\
\hline$P_{2}$ & 2 & $n$ & $n$ & $n$ \\
\hline$\overline{P_{3}}$ & 28 & $n+3$ & $n+3$ & $n+3$ \\
\hline$P_{4}$ & 32 & $n+4$ & $n+4$ & $n+4$ \\
\hline$P_{5}$ & 8 & $\begin{array}{l}n+4 \text { case } 1 \\
n+3 \text { case } 2\end{array}$ & $\begin{array}{l}n+6 \text { case } 1 \\
n+5 \text { case } 2 \\
n+4 \text { case } 3\end{array}$ & $\begin{array}{l}n+7 \text { case } 1 \\
n+6 \text { case } 2 \\
n+5 \text { case } 3\end{array}$ \\
\hline$S_{1,1,1}$ & 72 & 3 & 3 & 3 \\
\hline$S_{1,1,2}$ & 16 & 8 & 7 & 7 \\
\hline$C_{4}$ & 264 & 0 & 0 & 0 \\
\hline$L(4,1)$ & 112 & 0 & 0 & 0 \\
\hline$L(4,2)$ & 16 & 0 & 0 & 0 \\
\hline$C_{8}$ & 16 & $c_{8}$ & $\begin{array}{l}c_{8} \text { case } 1 \\
c_{8} \text { case } 2 \\
0 \text { case } 3\end{array}$ & $\begin{array}{c}c_{8} \text { case } 1 \\
c_{8} \text { case } 2 \\
0 \text { case } 3\end{array}$ \\
\hline \multicolumn{2}{|c|}{$\sum_{i} \lambda_{i}^{8}=$} & $\begin{array}{c}70 n+588+16 c_{8} \\
\text { for the case } 1 \\
70 n+580+16 c_{8} \\
\text { for the case } 2\end{array}$ & $\begin{array}{c}70 n+588+16 c_{8} \\
\text { for the case } 1 \\
70 n+580+16 c_{8} \\
\text { for the case } 2 \\
70 n+572 \\
\text { for the case } 3\end{array}$ & $\begin{array}{c}70 n+596+16 c_{8} \\
\text { for the case } 1 \\
70 n+588+16 c_{8} \\
\text { for the case } 2 \\
70 n+580 \\
\text { for the case } 3\end{array}$ \\
\hline
\end{tabular}

Table 4: Count of the motifs of some graphs $G \in \mathcal{G}_{3}$. We denote by $G_{a}\left(\operatorname{resp} . G_{b}, G_{c}\right)$ a graph described in proposition 20 (resp. 21, 22).

- case 2: $\exists r, s, t, r \neq s, r \neq t, s \neq t: d\left(u_{r}, u_{s}\right)=2$ and $d\left(u_{r}, u_{t}\right)>2$ and $d\left(u_{s}, u_{t}\right)>2$.

- case 3: $\forall s, t, d\left(u_{s}, u_{t}\right)>2$.

Then:

$$
\sum_{i} \lambda_{i}^{8}=\left\{\begin{array}{l}
70 n+596+16 c_{8} \text { for the case } 1 \\
70 n+588+16 c_{8} \text { for the case 2 } \\
70 n+580 \text { for the case } 3
\end{array}\right.
$$

and $G$ cannot be cospectral with a lollipop in the cases 2 and 3 and in the case 1 if $c_{8}=1$.

The two following propositions solve the case 1 of proposition 22 when $c_{8}=0$.

Proposition 23. Let $G \in \mathcal{G}_{3}$ such that $p \geq 10, \exists i, j, k: d\left(u_{i}, x_{i}\right)=d\left(u_{j}, x_{j}\right)=1$, $d\left(u_{k}, x_{k}\right)>2, d\left(u_{i}, u_{j}\right)=d\left(u_{j}, u_{k}\right)=2$. 
Then:

$$
\sum_{i} \lambda_{i}^{10}= \begin{cases}252 n+3340+20 c_{10} & \text { if } d\left(u_{k}, x_{k}\right)=3 \\ 252 n+3350+20 c_{10} & \text { if } d\left(u_{k}, x_{k}\right)>3\end{cases}
$$

where $c_{10}=\left|C_{10}(G)\right|$. And $G$ cannot be cospectral with $L(4, k)$.

Proof. We have $\left|P_{2}(G)\right|=n,\left|P_{3}(G)\right|=n+3,\left|P_{4}(G)\right|=n+4,\left|P_{5}(G)\right|=n+7$, $\left|P_{6}(G)\right|=n+6$ if $d\left(u_{k}, x_{k}\right)=3,\left|P_{6}(G)\right|=n+7$ if $d\left(u_{k}, x_{k}\right)>3,\left|S_{1,1,1}(G)\right|=3$, $\left|S_{1,1,2}(G)\right|=7,\left|S_{1,2,2}(G)\right|=5,\left|S_{1,1,3}(G)\right|=11$, and no others subgraphs $M$ such that $w_{k}(M)>0$. We then apply proposition 1 . The only case for which $\sum \lambda_{i}^{10}=252 n+3360$ is for the graph of figure 13, and we check that it is not cospectral with $L(4,11)$ by comparing spectral radii (see tables 11 and 12 in appendix).

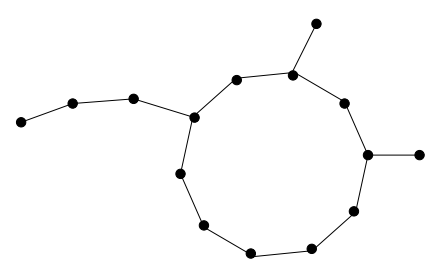

Figure 13:

Proposition 24. Let $G \in \mathcal{G}_{3}$ such that $p \geq 10, \exists i, j, k: d\left(u_{i}, x_{i}\right)=d\left(u_{j}, x_{j}\right)=1$, $d\left(u_{k}, x_{k}\right)>2, d\left(u_{i}, u_{k}\right)=d\left(u_{j}, u_{k}\right)=2$. Then $G$ cannot be cospectral with $L(4, k)$.

Proof. Let $G$ be a graph cospectral with $L(4, k)$ and let $q=d\left(u_{k}, x_{k}\right)$ (we have $n=p+q+2)$. Applying theorem 5 to the vertex $u_{k}$, we have:

$$
\begin{aligned}
Q_{G}(X)= & X Q_{T_{p+1}}(X) Q_{P_{q}}(X)-2 Q_{S_{1,1, p-3}}(X) Q_{P_{q}}(X) \\
& -Q_{T_{p+1}}(X) Q_{P_{q-1}}(X)-2 X^{2} Q_{P_{q}}(X)
\end{aligned}
$$

Property 2 gives $Q_{G}(2)=-16(q+1)$ and according to proposition $5 G$ is cospectral with a lollipop $L(4, k)$ only if $-16(q+1)=-4 n+16$ ie $p=3 q+6$ and $q$ is necessarily even.

Using $Q_{S_{1,1, c}}(X)=X\left(Q_{P_{c+2}}(X)-Q_{P_{c}}(X)\right)$ we have that if $c$ is odd then 0 is an eigenvalue of $S_{1,1, c}$ with multiplicity 2 and if $R(X)=\frac{Q_{S_{1,1, c}}(X)}{X^{2}}$ then $R(0)=(-1)^{\frac{c+1}{2}}(c+2)$. The relation $Q_{T_{n}}(X)=X Q_{S_{1,1, n-4}}(X)-X Q_{S_{1,1, n-6}}(X)$ implies that 0 is an eigenvalue of $T_{n}$ with multiplicity 2.

Let $R(X)=\frac{Q_{G}(X)}{X^{2}}$. Property 4 gives

$$
R(0)=\left\{\begin{aligned}
-2 p & \text { if } q \equiv 0[4] \\
-2 p+4 & \text { if } q \equiv 2[4]
\end{aligned}\right.
$$


If $q \equiv 0[4]$ then according to proposition $6, G$ is cospectral with a lollipop $L(4, k)$ only if $-2 p=-n$ ie $p=q+2$ which contradicts $p=3 q+6$.

If $q \equiv 2[4]$ then according to proposition $6, G$ is cospectral with a lollipop $L(4, k)$ only if $-2 p+4=-n$ ie $p=q+6$ which contradicts $p=3 q+6$.

\subsection{Case IV: Graphs with $n_{4}=1, n_{3}=0 n_{1}=2, n_{0}=0, c=0$ and}

$$
n_{2}=n-3
$$

The graph $\gamma_{p, k_{1}, k_{2}}$ is the coalescence of a lollipop $L\left(p, k_{1}\right)$ with the vertex of degree 3 as distinguished vertex and a path $P_{k_{2}+1}$ with a pendant vertex as distinguished vertex (cf figure 14 for an example). A connected graph with $n_{4}=1, n_{3}=0 n_{1}=2, n_{0}=0$ and $n_{2}=n-3$ is a $\gamma_{p, k_{1}, k_{2}}$.

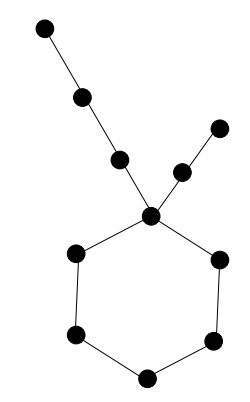

Figure 14: $\gamma_{6,2,3}$

Proposition 25. For a graph $\gamma_{p, k_{1}, k_{2}}$ with $p>4$ we have:

$$
\sum_{i} \lambda_{i}^{6}=\left\{\begin{array}{l}
20 n+96+12 c_{6} \quad \text { if } k_{1}=k_{2}=1 \\
20 n+108+12 c_{6} \quad \text { if } k_{1}>1, k_{2}=1 \\
20 n+120+12 c_{6} \quad \text { if } k_{1}>1, k_{2}>1
\end{array}\right.
$$

where $c_{6}=\left|C_{6}(G)\right|$.

Proof. We have $\left|P_{2}(G)\right|=n,\left|P_{3}(G)\right|=n+3,\left|S_{1,1,1}(G)\right|=4$ and

- $\left|P_{4}(G)\right|=n+2$ if $k_{1}=k_{2}=1$

- $\left|P_{4}(G)\right|=n+4$ if $k_{1}>k_{2}=1$

- $\left|P_{4}(G)\right|=n+6$ if $k_{1} \geq k_{2}>1$

and we apply proposition 1. 
Proposition 26. A lollipop $L(4, k)$ cannot be cospectral with a graph $\gamma_{p, 1,1}$.

Proof. The graphs $L(4, k)$ and $\gamma_{p, 1,1}$ have $n=k+4=p+2$ vertices. Let us show that $Q_{L(4, k)}(2) \neq P_{\gamma_{p, 1,1}}(2)$. Using twice the theorem 6:

$$
\begin{aligned}
P_{\gamma_{p, 1,1}}(X) & =X Q_{L(p, 1)}(X)-X Q_{P_{p-1}}(X) \\
& =X\left(X Q_{C_{p}}(X)-Q_{P_{p-1}}(X)\right)-X Q_{P_{p-1}}(X)
\end{aligned}
$$

And by property $2, P_{\gamma_{p, 1,1}}(2)=-4 p=-4 n+8$ which contradicts $Q_{L(4, k)}(2)=-4 n+16$ (proposition 5).

Theorem 22. A lollipop $L(4, k)$ cannot be cospectral with $\gamma_{p, k_{1}, k_{2}}, p>4$.

Proof. It is a straightforward consequence of propositions 3,25 and 26.

\section{Conclusion}

In this paper we give a way to count closed walks, which is relevant to show that two graphs cannot be cospectral and that provides a new approach to show that there are no cospectral non-isomorphic lollipops. We then show that the lollipop graph is determined by its spectrum. We first give a proof, based on computing the degree distribution, for lollipops $L(p, k)$ with $p \neq 4$. The most difficult case, as it was noted in [8,3], is for the lollipops $L(4, k)$; we solved this case following the same idea as the general case. Showing connectivity of a graph cospectral with a $L(4, k)$ and non-cospectrality of a given graph with a $L(4, k)$ are both quite long to establish and have required the use of a large variety of tools.

\section{Acknowledgements}

We thank an anonymous referee for bringing the sketch of the Tayfeh-Rezaie's proof of theorem 16 to our attention. 


\section{A Appendix}

\section{A.1 Counting covering closed walks}

\begin{tabular}{|c|c|c|c|}
$M$ & $w_{6}(M)$ & $w_{8}(M)$ & $w_{10}(M)$ \\
\hline$P_{2}$ & 2 & 2 & 2 \\
$P_{3}$ & 12 & 28 & 60 \\
$P_{4}$ & 6 & 32 & 120 \\
$P_{5}$ & 0 & 8 & 60 \\
$P_{6}$ & 0 & 0 & 10 \\
$C_{4}$ & 48 & 264 & 1320 \\
$C_{6}$ & 12 & & \\
$C_{8}$ & 0 & 16 & \\
$C_{10}$ & 0 & 0 & 20 \\
$S_{1,1,1}$ & 12 & 72 & 300 \\
$S_{1,1,2}$ & 0 & 16 & 140 \\
$S_{1,1,3}$ & 0 & 0 & 20 \\
$S_{1,2,2}$ & 0 & 0 & 20 \\
$L(4,1)$ & 12 & 112 & 840 \\
$L(4,2)$ & 0 & 16 & 180 \\
$L(4,3)$ & 0 & 0 & 20 \\
\hline
\end{tabular}

Table 5: Number of covering closed walks on a given graph.

\section{A.2 Proof of theorem 17}

First, we notice the following relations which will be useful to prove lemmas 3 and 6 and whose proof is straightfoward by induction on $p$.

$$
\forall p>0, Q_{P_{p}}(\alpha)>\beta Q_{P_{p-1}}(\alpha)
$$

where $\alpha=\sqrt{2+2 \sqrt{2}}$ and $\beta=\frac{\sqrt{2}}{2} \alpha$. Obviously equation 2 is true if we replace $\beta$ by $\beta^{\prime} \leq \beta$.

Lemma 2. $\lambda_{1}\left(P\left(p_{1}, p_{2}, p_{3}\right)\right)>2$.

Proof. On one hand $\lambda_{1}(P(0,1,1))>2$ and $\lambda_{1}(P(1,1,1))>2$. On the other hand, if there exists $p_{i} \geq 2$ (we assume $p_{3} \geq 2$ ) then the lollipop $L\left(p_{1}+p_{2}+2,1\right)$ is an induced subgraph of $P\left(p_{1}, p_{2}, p_{3}\right)$. Since $\lambda_{1}\left(L\left(p_{1}+p_{2}+2,1\right)\right)>2$ (theorem 11) the interlacing theorem gives the result. 
Applying theorem 5 to a vertex of degree 3 of $P\left(p_{1}, p_{2}, p_{3}\right)$ we can get the following expression of the characteristic polynomial of $P\left(p_{1}, p_{2}, p_{3}\right), p_{i}>0$ which will be useful for the next results.

$$
\begin{array}{r}
Q_{P\left(p_{1}, p_{2}, p_{3}\right)}(X)=X Q_{S_{p_{1}, p_{2}, p_{3}}}(X)-Q_{S_{p_{1}-1, p_{2}, p_{3}}}(X)-Q_{S_{p_{1}, p_{2}-1, p_{3}}}(X) \\
-Q_{S_{p_{1}, p_{2}, p_{3}-1}}(X)-2 Q_{P_{p_{1}}}(X)-2 Q_{P_{p_{2}}}(X)-2 Q_{P_{p_{3}}}(X)
\end{array}
$$

where

$$
\begin{aligned}
Q_{S_{a, b, c}}(X) & =X Q_{P_{a}}(X) Q_{P_{b}}(X) Q_{P_{c}}(X)-Q_{P_{a-1}}(X) Q_{P_{b}}(X) Q_{P_{c}}(X) \\
& -Q_{P_{a}}(X) Q_{P_{b-1}}(X) Q_{P_{c}}(X)-Q_{P_{a}}(X) Q_{P_{b}}(X) Q_{P_{c-1}}(X)
\end{aligned}
$$

Lemma 3. If $p_{1} \leq 3, p_{2} \leq 3$ then $\forall p \in \mathbb{N}: \lambda_{1}\left(P\left(p_{1}, p_{2}, p\right)\right)>\sqrt{2+2 \sqrt{2}}$.

Proof. According to theorem 7 it is sufficient to prove the result for $p_{1}=3, p_{2}=3$. Let $\alpha=\sqrt{2+2 \sqrt{2}}$. We shall show that $Q_{P(3,3, p)}(\alpha)<0$. Using equations (3) and (4) and $Q_{P_{p-2}}(X)=X Q_{P_{p-1}}(X)-Q_{P_{p}}(X), Q_{P_{2}}(X)=X^{2}-1, Q_{P_{3}}(X)=X^{3}-2 X$ and $Q_{P_{4}}(X)=X^{4}-3 X^{2}+1$, we get:

$$
\begin{aligned}
Q_{P(3,3, p)}(X)= & Q_{P_{p}}(X)\left(X^{8}-9 X^{6}+24 X^{4}-20 X^{2}\right) \\
& +Q_{P_{p-1}}(X)\left(-X^{7}+8 X^{5}-16 X^{3}+8 X\right)-4\left(X^{3}-2 X\right)
\end{aligned}
$$

so

$$
\begin{aligned}
Q_{P(3,3, p)}(\alpha) & =(16-16 \sqrt{2}) Q_{P_{p}}(\alpha)+\alpha(16-8 \sqrt{2}) Q_{P_{p-1}}(\alpha)-8 \sqrt{2} \alpha \\
& =(-16+16 \sqrt{2})\left(-Q_{P_{p}}(\alpha)+\frac{\alpha}{\sqrt{2}} Q_{P_{p-1}}(\alpha)\right)-8 \sqrt{2} \alpha<0 \text { by }(2)
\end{aligned}
$$

As a result $\lambda_{1}(P(3,3, p))>\alpha$.

Lemma 4. If $p_{1} \leq 2, p_{2} \leq 4$ then $\forall p \in \mathbb{N}: \lambda_{1}\left(P\left(p_{1}, p_{2}, p\right)\right)>2.2>\sqrt{2+2 \sqrt{2}}$

Proof. Mutatis mutandis the proof is the same as the one of lemma 3.

Lemma 5. For $p_{2}, p_{3}>0, p_{1} \in\{0,1\}$ we have $\lambda_{1}\left(P\left(p_{1}, p_{2}, p_{3}\right)\right)>\sqrt{2+2 \sqrt{2}}$.

Proof. Let $\alpha=\sqrt{2+2 \sqrt{2}}$. According to theorem 7 it is sufficient to prove the result for $P(1, p, p)$ where $p=\max \left(p_{2}, p_{3}\right)$. Applying theorem 5 to the vertex at distance one of the two vertices of degree 3 we have:

$$
\begin{aligned}
Q_{P(1, p, p)}(X) & =X Q_{C_{2 p+2}}(X)-2 Q_{P_{2 p+1}}(X)-4 Q_{P_{p}}(X) \\
& =X\left(X Q_{P_{2 p+1}}(X)-2 Q_{P_{2 p}}(X)-2\right)-2 Q_{P_{2 p+1}}(X)-4 Q_{P_{p}}(X) \\
& =\left(X^{2}-2\right) Q_{P_{2 p+1}}(X)-2 X Q_{P_{2 p}}(X)-4 Q_{P_{p}}(X)-2 X
\end{aligned}
$$


But (theorem 5 applied to a vertex at distance $p$ of a pendant vertex in the graphs $P_{2 p+1}$ and $\left.P_{2 p}\right)$ :

$$
Q_{P_{2 p+1}}(X)=X\left(Q_{P_{p}}^{2}(X)\right)-2 Q_{P_{p}}(X) Q_{P_{p-1}}(X)
$$

and

$$
Q_{P_{2 p}}(X)=X Q_{P_{p}}(X) Q_{P_{p-1}}(X)-Q_{P_{p-1}}^{2}(X)-Q_{P_{p}}(X) Q_{P_{p-2}}(X) .
$$

So

$$
\begin{aligned}
Q_{P(1, p, p)}(X)= & \left(X^{2}-2\right)\left(X Q_{P_{p}}^{2}(X)-2 Q_{P_{p}}(X) Q_{P_{p-1}}(X)\right) \\
& -2 X\left(X Q_{P_{p}}(X) Q_{P_{p-1}}(X)-Q_{P_{p-1}}^{2}(X)-Q_{P_{p}}(X) Q_{P_{p-2}}(X)\right) \\
& -4 Q_{P_{p}}(X)-2 X \\
= & Q_{P_{p}}(X)\left(\left(X^{3}-4 X\right) Q_{P_{p}}(X)+\left(4-2 X^{2}\right) Q_{P_{p-1}}(X)-4\right) \\
& +2 X Q_{P_{p-1}}^{2}(X)-2 X
\end{aligned}
$$

Using $Q_{P_{p}}(\alpha)>\beta Q_{P_{p-1}}(\alpha)$ (equation (2)), we get

$$
Q_{P(1, p, p)}(\alpha)<Q_{P_{p}}(\alpha)\left(\left(\alpha^{3}-4 \alpha\right) Q_{P_{p}}(\alpha)+\left(4-2 \alpha^{2}+\frac{2 \alpha}{\beta}\right) Q_{P_{p-1}}(\alpha)-4\right)-2 \alpha .
$$

We then notice that $\frac{4-2 \alpha^{2}+\frac{2 \alpha}{\beta}}{-\alpha^{3}+4 \alpha}=\beta$ and by equation (2) we have $Q_{P(1, p, p)}(\alpha)<0$.

Lemma 6. Given $P\left(2, p_{2}, p_{3}\right)$ with $p_{3} \geq 3$, denote by $u$ and $v$ the two vertices of degree 3. Let $y$ be a vertex at distance 2 from $u$ and at distance greater than or equal to 2 from $v$, we define $\tilde{P}\left(2, p_{2}, p_{3}\right)$ as the graph obtained by adding to $P\left(2, p_{2}, p_{3}\right)$ a pendant vertex $x$ to $y$. We have $\lambda_{1}\left(\tilde{P}\left(2, p_{2}, p_{3}\right)\right)>\sqrt{2+2 \sqrt{2}}$.

Proof. Let $\alpha=\sqrt{2+2 \sqrt{2}}$. By theorem 7 it is sufficient to prove the result for $p_{2}=p_{3}=p=\max \left\{p_{2}, p_{3}\right\}$. The aim of the proof is to show that $Q_{\tilde{P}(2, p, p)}(\alpha)<0$. The following equations will be useful:

$$
\begin{gathered}
Q_{S_{2, a, b}}(\alpha)=\left(\alpha^{2}-1\right) Q_{P_{a+b+1}}(\alpha)-\alpha Q_{P_{a}}(\alpha) Q_{P_{b}}(\alpha) \\
Q_{P_{2 p+1}}(\alpha)=\alpha Q_{P_{p}}^{2}(\alpha)-2 Q_{P_{p}}(\alpha) Q_{P_{p-1}}(\alpha) \\
Q_{P_{2 p}}(\alpha)=Q_{P_{p}}^{2}(\alpha)-Q_{P_{p-1}}^{2}(\alpha) \\
Q_{P_{2 p-1}}(\alpha)=\alpha Q_{P_{2 p}}(\alpha)-Q_{P_{2 p+1}}(\alpha)=-\alpha Q_{P_{p-1}}^{2}(\alpha)+2 Q_{P_{p}}(\alpha) Q_{P_{p-1}}(\alpha)
\end{gathered}
$$

and we deduce

$$
\begin{gathered}
Q_{S_{2, p, p}}(\alpha)=\left(\alpha^{3}-2 \alpha\right) Q_{P_{p}}^{2}(\alpha)-2\left(\alpha^{2}-1\right) Q_{P_{p}}(\alpha) Q_{P_{p-1}}(\alpha) \\
Q_{S_{2, p, p-1}}(\alpha)=\left(\alpha^{2}-1\right) Q_{P_{p}}^{2}(\alpha)-\left(\alpha^{2}-1\right) Q_{P_{p-1}}^{2}(\alpha)-\alpha Q_{P_{p}}(\alpha) Q_{P_{p-1}}(\alpha) \\
Q_{S_{2, p+1, p-1}}(\alpha)=\left(\alpha^{3}-\alpha\right) Q_{P_{p}}^{2}(\alpha)+\left(-3 \alpha^{2}+2\right) Q_{P_{p}}(\alpha) Q_{P_{p-1}}(\alpha)+\alpha Q_{P_{p-1}}^{2}(\alpha)
\end{gathered}
$$


Theorem 5 gives

$$
Q_{\tilde{P}(2, p, p)}(\alpha)=\alpha Q_{P(2, p, p)}(\alpha)-Q_{H}(\alpha)
$$

where $H=\tilde{P}(2, p, p) \backslash\{x, y\}$ Equation 3 gives

$$
Q_{P(2, p, p)}(\alpha)=\alpha Q_{S_{2, p, p}}(\alpha)-Q_{S_{1, p, p}}(\alpha)-2 Q_{S_{2, p, p-1}}(\alpha)-4 Q_{P_{p}}(\alpha)-2 Q_{P_{2}}(\alpha)
$$

but $Q_{S_{1, p, p}}(\alpha)=\frac{1}{\alpha}\left(Q_{S_{2, p, p}}(\alpha)+Q_{S_{0, p, p}}(\alpha)\right)$ so

$$
\begin{aligned}
\alpha Q_{P(2, p, p)}(\alpha)= & \left(\alpha^{2}-1\right) Q_{S_{2, p, p}}(\alpha)-2 \alpha Q_{S_{2, p, p-1}}(\alpha)-Q_{P_{2 p+1}}(\alpha) \\
& -4 \alpha Q_{P_{p}}(\alpha)-2 \alpha Q_{P_{2}}(\alpha) \\
= & \left(\alpha^{5}-5 \alpha^{3}+3 \alpha\right) Q_{P_{p}}^{2}(\alpha)+\left(2 \alpha^{3}-2 \alpha\right) Q_{P_{p-1}}^{2}(\alpha) \\
& +\left(-2 \alpha^{4}+6 \alpha^{2}\right) Q_{P_{p}}(\alpha) Q_{P_{p-1}}(\alpha)-4 \alpha Q_{P_{p}}(\alpha)-2 \alpha Q_{P_{2}}(\alpha)
\end{aligned}
$$

Theorem 5 gives

$$
Q_{H}(\alpha)=\alpha^{2} Q_{S_{2, p, p-2}}(\alpha)-\alpha Q_{S_{1, p, p-2}}(\alpha)-\alpha Q_{S_{2, p-1, p-2}}(\alpha)-Q_{S_{2, p, p-2}}(\alpha)-2 \alpha Q_{P_{p-2}}(\alpha)
$$

but $Q_{S_{2, p, p-2}}(\alpha)=\alpha Q_{S_{2, p, p-1}}(\alpha)-Q_{S_{2, p, p}}(\alpha), \alpha Q_{S_{1, p, p-2}}(\alpha)=Q_{S_{2, p, p-2}}(\alpha)+Q_{S_{0, p, p-2}}(\alpha)$ and $Q_{S_{2, p-1, p-2}}(\alpha)=\left(\alpha^{2}-1\right) Q_{S_{2, p, p-1}}(\alpha)-\alpha Q_{S_{2, p-1, p+1}}(\alpha)$ so

$$
\begin{aligned}
Q_{H}(\alpha)= & -\alpha Q_{S_{2, p, p-1}}(\alpha)-\left(\alpha^{2}-2\right) Q_{S_{2, p, p}}(\alpha)+\alpha^{2} Q_{S_{2, p-1, p+1}}(\alpha) \\
& -Q_{P_{2 p-1}}(\alpha)-2 \alpha Q_{P_{p-2}}(\alpha) \\
= & \left(2 \alpha^{3}-3 \alpha\right) Q_{P_{p}}^{2}(\alpha)+\left(2 \alpha^{3}\right) Q_{P_{p-1}}^{2}(\alpha) \\
& +\left(-\alpha^{4}-3 \alpha^{2}+2\right) Q_{P_{p}}(\alpha) Q_{P_{p-1}}(\alpha)-2 \alpha Q_{P_{p-2}}(\alpha)
\end{aligned}
$$

So we have:

$$
\begin{aligned}
Q_{\tilde{P}(2, p, p)}(\alpha)= & \left(\alpha^{5}-7 \alpha^{3}+6 \alpha\right) Q_{P_{p}}^{2}(\alpha)-2 \alpha Q_{P_{p-1}}^{2}(\alpha) \\
& +\left(-\alpha^{4}+9 \alpha^{2}-2\right) Q_{P_{p}}(\alpha) Q_{P_{p-1}}(\alpha) \\
& +2 \alpha Q_{P_{p-2}}(\alpha)-4 \alpha Q_{P_{p}}(\alpha)-2 \alpha Q_{P_{2}}(\alpha)
\end{aligned}
$$

Equation (2) gives $2 \alpha Q_{P_{p-2}}(\alpha)-4 \alpha Q_{P_{p}}(\alpha)<0$.

Lets us show that $x Q_{P_{p}}^{2}(\alpha)+y Q_{P_{p-1}}^{2}(\alpha)+z Q_{P_{p}}(\alpha) Q_{P_{p-1}}(\alpha)<0$ with $x=\alpha^{5}-7 \alpha^{3}+6 \alpha$, $y=-2 \alpha, z=-\alpha^{4}+9 \alpha^{2}-2$. Note that $\frac{y}{z+\beta x}=-\beta$, where $\beta$ is defined in equation (2).

$$
\begin{array}{r}
x Q_{P_{p}}^{2}(\alpha)+y Q_{P_{p-1}}^{2}(\alpha)+z Q_{P_{p}}(\alpha) Q_{P_{p}}(\alpha)= \\
Q_{P_{p}}(\alpha)\left(x Q_{P_{p}}(\alpha)-\beta x Q_{P_{p-1}}(\alpha)\right)+Q_{P_{p-1}}(\alpha)\left((z+\beta x) Q_{P_{p}}(\alpha)+y Q_{P_{p-1}}(\alpha)\right)= \\
Q_{P_{p}}(\alpha) x\left(Q_{P_{p}}(\alpha)-\beta Q_{P_{p-1}}(\alpha)\right)+Q_{P_{p-1}}(\alpha)(z+\beta x)\left(Q_{P_{p}}(\alpha)-\beta Q_{P_{p-1}}(\alpha)\right)<0
\end{array}
$$

because $\frac{(z+\beta x)\left(Q_{P_{p}}(\alpha)-\beta Q_{P_{p-1}}(\alpha)\right)}{-x\left(Q_{P_{p}}(\alpha)-\beta Q_{P_{p-1}}(\alpha)\right)}=\frac{z+\beta x}{-x}<\beta$ and we use equation (2). 
Lemma 7. Given $P\left(2, p_{2}, p_{3}\right)$ with $p_{3} \geq 3$, denote by $u$ and $v$ the two vertices of degree 3. Let $y$ be a vertex at distance 1 from $u$ and at distance greater than or equal to 1 from $v$, we denote by $\hat{P}\left(2, p_{2}, p_{3}\right)$ the graph obtained by adding to $P\left(2, p_{2}, p_{3}\right)$ a pendant vertex $x$ to $y$. We have $\lambda_{1}\left(\hat{P}\left(2, p_{2}, p_{3}\right)\right)>\sqrt{2+2 \sqrt{2}}$.

Proof. A direct consequence of theorem 7 and lemma 6.

Theorem 17. For $p_{1}, p_{2}, p_{3}>0, P\left(p_{1}, p_{2}, p_{3}\right)$ cannot be an induced subgraph of a graph cospectral with a $L(4, k)$.

Proof. Without loss of generality we assume $p_{1} \leq p_{2} \leq p_{3}$. In order to lead a proof by contradiction, let $P\left(p_{1}, p_{2}, p_{3}\right)$ be an induced subgraph of $G$ cospectral with a $L(4, k)$. As $G$ is bipartite, $P\left(p_{1}, p_{2}, p_{3}\right)$ doesn't have odd cycles and the $p_{i}$ 's are all odd or all even. Using equation (3) and property 2 we obtain:

$$
Q_{P\left(p_{1}, p_{2}, p_{3}\right)}(2)=p_{1} p_{2} p_{3}-p_{1} p_{2}-p_{1} p_{3}-p_{2} p_{3}-3 p_{1}-3 p_{2}-3 p_{3}-5
$$

i) First assume that $p_{1}, p_{2}, p_{2}$ are odd.

By lemma 5 we have $p_{1} \geq 3$ and by lemma 3 we have $p_{2} \geq 5$.

- If $p_{1}=3$ and $p_{2}=5$ then $Q_{P\left(p_{1}, p_{2}, p_{3}\right)}(2)=4 p_{3}-44 \geq 0$ if $p_{3} \geq 11$

- If $p_{1}=3$ and $p_{2} \geq 7$ then $Q_{P\left(p_{1}, p_{2}, p_{3}\right)}(2) \geq 2 p_{3}-14 \geq 0$ (because $p_{3} \geq p_{2} \geq 7$ )

- If $5 \leq p_{1} \leq p_{2} \leq p_{3}$ then $Q_{P\left(p_{1}, p_{2}, p_{3}\right)}(2) \geq p_{3}-5 \geq 0$.

$Q_{P\left(p_{1}, p_{2}, p_{3}\right)}(2) \geq 0$ implies that $P\left(p_{1}, p_{2}, p_{3}\right)$ has two eigenvalues greater than or equal to 2 (we already know by lemma 2 that $P\left(p_{1}, p_{2}, p_{3}\right)$ has at least one eigenvalue strictly greater than 2) and since a lollipop has only one eigenvalue greater than 2 (theorem 11 ), the interlacing theorem provides a contradiction except when $p_{1}=3, p_{2}=5$ and $p_{3} \in\{5,7,9\}$.

According to tables 7 and $11, P\left(3,5, p_{3}\right)$ cannot be a connected component of a graph cospectral with $L(4, k)$ because $\lambda_{1}\left(P\left(3,5, p_{3}\right)\right)<2.195$ while $\lambda_{1}(L(4, k)) \geq \lambda_{1}(L(4,5))>$ 2.195 when $k \geq 5$. So there is a new vertex $x$ adjacent to one vertex $y$ of $P\left(3,5, p_{3}\right)$ (and only one because otherwise there exists $r, s \in \mathbb{N}$ such that $P(1, r, s)$ is an induced subgraph of $G$ which is impossible by lemma 5$)$. Let $H$ be the subgraph induced by $P\left(3,5, p_{3}\right)$ and $x$, denote by $u$ and $v$ the two vertices of degree 3 in $P\left(3,5, p_{3}\right)$.

1. If $y=u$ or $y=v$ then the graph $T$ drawn on figure 15 is an induced subgraph of $H$ and $\lambda_{1}(T) \geq 2.20>\sqrt{2+2 \sqrt{2}}>\lambda_{1}(L(p, k))$ and $H$ cannot be an induced subgraph of $G$.

2. If $\min \{d(y, u), d(y, v)\} \geq 5$ the disjoint union of a cycle and $S_{1,3,3}$ is an induced subgraph of $H$ with twice the eigenvalue 2, so $H$ cannot be an induced subgraph of $G$ (by the interlacing theorem and theorem 11). 


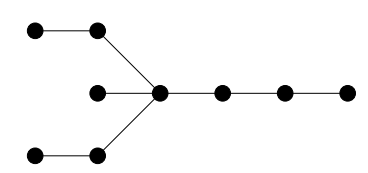

Figure 15: Tree $T$ whose spectral radius is greater than 2.20

3. The cases where $1 \leq \min \{d(y, u), d(y, v)\} \leq 4$ are summed up in table 9 . For all these cases $H$ cannot be an induced subgraph of $G$ because either $H$ has two eigenvalues greater than 2 or $H$ has a spectral radius greater than $\sqrt{2+2 \sqrt{2}}$.

As a result $P\left(p_{1}, p_{2}, p_{3}\right)$ with $p_{i}$ 's odd cannot be an induced subgraph of $G$.

ii) We now assume that $p_{1}, p_{2}, p_{3}$ are even.

By lemma 5 we have $p_{1} \geq 2$.

- If $p_{1}=2$ and $p_{2} \leq 4$ then by lemma $4 P\left(p_{1}, p_{2}, p_{3}\right)$ cannot be an induced subgraph of $G$.

- If $p_{1}=2$ and $p_{2}=6$ then $Q_{P\left(p_{1}, p_{2}, p_{3}\right)}(2)=p_{3}-41 \geq 0$ if $p_{3} \geq 42$

- If $p_{1}=2$ and $p_{2}=8$ then $Q_{P\left(p_{1}, p_{2}, p_{3}\right)}(2)=3 p_{3}-51 \geq 0$ if $p_{3} \geq 18$

- If $p_{1}=2$ and $p_{2}=10$ then $Q_{P\left(p_{1}, p_{2}, p_{3}\right)}(2)=5 p_{3}-61 \geq 0$ if $p_{3} \geq 14$

- If $p_{1}=2$ and $p_{2} \geq 12$ then $Q_{P\left(p_{1}, p_{2}, p_{3}\right)}(2) \geq 2 p_{3}-11 \geq 0$ (because $\left.p_{3} \geq 12\right)$

- If $p_{1}=4$ and $p_{2}=4$ then $Q_{P\left(p_{1}, p_{2}, p_{3}\right)}(2)=5 p_{3}-45 \geq 0$ if $p_{3} \geq 10$

- If $p_{1}=4$ and $p_{2} \geq 6$ then $Q_{P\left(p_{1}, p_{2}, p_{3}\right)}(2) \geq 4 p_{3}-17 \geq 0$ (because $p_{3} \geq 6$ )

- If $6 \leq p_{1} \leq p_{2} \leq p_{3}$ then $Q_{P\left(p_{1}, p_{2}, p_{3}\right)}(2) \geq 9 p_{3}-5 \geq 0$.

As in the proof of the odd case, if $Q_{P\left(p_{1}, p_{2}, p_{3}\right)}(2) \geq 0$ then $P\left(p_{1}, p_{2}, p_{3}\right)$ has two eigenvalues greater than or equal to 2 and cannot be an induced subgraph of $G$. We are now going to study the remaining cases for $p_{1}=2$ and $p_{1}=4$.

First case $p_{1}=2$ :

The only unsolved cases we are going to consider here are for $p_{2} \in\{6,8,10\}$ with the corresponding constraints on $p_{3}$. As it was detailed in the proof of the odd case, none of these graphs is a connected component of a graph cospectral with $L(4, k)$ and so there is a new vertex $x$ adjacent to one and only one vertex $y$ of $P\left(2, p_{2}, p_{3}\right)$. Let $H$ be the subgraph induced by $P\left(2, p_{2}, p_{3}\right)$ and $x$. With the same notations and arguments as for the odd case, $H$ cannot be an induced subgraph of $G$ when $\min \{d(y, u), d(y, v)\} \geq 5$ or $y=u$ or

$y=v$. Moreover if $\min \{d(y, u), d(y, v)\} \leq 2$ then by lemmas 7 and $6, \lambda_{1}(H)>\sqrt{2+2 \sqrt{2}}$ so $H$ cannot be an induced subgraph of a lollipop. We are now going to examine the two last tricky cases: $\min \{d(y, u), d(y, v)\}=3$ and $\min \{d(y, u), d(y, v)\}=4$. 
- If $\min \{d(y, u), d(y, v)\}=3$, we can assume that $d(y, v)=3$. Let $\{b, c\}=\left\{p_{2}, p_{3}\right\}$ such that $y$ is a vertex belonging to a path of length $c+1$ of $P(2, b, c)$ between $u$ and $v$. Then applying theorem 6 to $x$ we get $Q_{H}(X)=X Q_{P(2, b, c)}(X)-Q_{P(2, b, c) \backslash\{y\}}(X)$ and applying theorem 5 to $v$ we have:

$$
\begin{aligned}
Q_{P(2, b, c) \backslash\{y\}}(X)= & X Q_{P_{2}}(X) Q_{S_{2, b, c-3}}(X)-Q_{P_{2}}(X) Q_{S_{1, b, c-3}}(X) \\
& -Q_{P_{2}}(X) Q_{S_{2, b-1, c-3}}(X)-Q_{P_{1}}(X) Q_{S_{2, b, c-3}}(X) \\
& -2 Q_{P_{2}}(X) Q_{P_{c-3}}(X)
\end{aligned}
$$

Using equation (3) and property 2 which gives the value in 2 of the characteristic polynomials of paths and $T$-shape trees we obtain $Q_{H}(2)=b c-5 b+4 c-56$.

- If $b \leq c$

* If $b=6$ (so $c \geq 6$ ) then $Q_{H}(2)=10 c-86$ so if $c \geq 10, H$ has two eigenvalues greater than 2 and cannot be and induced subgraph of $G$. Otherwise for $c=8$ we check that $\lambda_{1}(H) \sim 2.2050>\sqrt{2+2 \sqrt{2}}$ and so $H$ cannot be an induced subgraph of $G$ for $c \leq 8$.

* If $c \geq b \geq 8$ then $Q_{H}(2) \geq 7 c-56 \geq 0$ and $H$ has two eigenvalues greater than 2 and cannot be an induced subgraph of $G$.

- If $b \geq c$

* If $c=6$ then $Q_{H}(2)=b-32$ so if $b \geq 32$ then $H$ has two eigenvalues greater than 2 and cannot be an induced subgraph of $G$. Otherwise we check that for $b=30$ we have $\lambda_{1}(H) \sim 2.2071>\sqrt{2+2 \sqrt{2}}$ and so $H$ cannot be an induced subgraph of $G$ for $b \leq 30$.

* If $8 \leq c \leq b$ then $Q_{H}(2) \geq 4 c-32 \geq 0$ and $H$ has two eigenvalues greater than 2 and cannot be an induced subgraph of $G$.

- If $\min \{d(y, u), d(y, v)\}=4$, note that $c \geq 8$ (otherwise $y$ is at distance less than 4 from $u$ or $v$ ). In the same way as previously we compute $Q_{H}(2): Q_{H}(2)=b+9 c-86$.

- If $b \leq c$

* If $b=6$ then $Q_{H}(2)=9 c-80$. So if $c \geq 10$ then $H$ has two eigenvalues greater than 2 and cannot be an induced subgraph of $G$. Otherwise we check that for $c=8$ we have $\lambda_{1}(H) \sim 2.2014>\sqrt{2+2 \sqrt{2}}$.

* If $b=8$ then $Q_{H}(2)=9 c-78$. So if $c \geq 10$ then $H$ has two eigenvalues greater than 2 and cannot be an induced subgraph of $G$. The case $c=b=8$ is considered further in the proof.

* If $10 \leq b \leq c$ then $Q_{H}(2)>0$ and $H$ has two eigenvalues greater than 2 and cannot be an induced subgraph of $G$.

- If $b \geq c$ 
* If $c=8$ then $Q_{H}(2)=b-14$. So if $b \geq 14$ then $H$ has two eigenvalues greater than 2 and cannot be an induced subgraph of $G$. Otherwise we check for $c=8$ and $8 \leq b \leq 12$ that $\lambda_{1}(H)<2.196$ so $H$ cannot be a connected component of $G$ because for $k \geq 6 \lambda_{1}(L(4, k)) \geq \lambda_{1}(L(4,6))>$ 2.196. And so there is a new vertex $x^{\prime}$ adjacent to a vertex $y^{\prime}$ of $H$. Let $H^{\prime}$ be the graph induced by $H$ and $x^{\prime}$.

- If $y^{\prime}=y$ then $x^{\prime}$ is not adjacent to another vertex of $P(2, a, b)$ otherwise there exists $r, s \in \mathbb{N}$ such that $P(1, r, s)$ is an induced subgraph of $G$ which is impossible by lemma 5 and $x^{\prime}$ is not adjacent to $x$ otherwise $G$ contains a triangle (impossible because $G$ is bipartite). Hence $x^{\prime}$ is a pendant in $H^{\prime}$. The graph $H^{\prime}$ then contains $C_{q} \cup S_{4}$ (for $q \geq 3$ ) as an induced subgraph and so has two eigenvalues greater than 2 which is impossible.

- Assume that $y^{\prime}=x$. If $x^{\prime}$ is adjacent to another vertex $z$ of $H$ distinct from $y^{\prime}$ and $y$, then by the previous cases we necessarily have $\min \{d(z, u), d(z, v)\}=4$. Either the graph $S_{1,3,3} \cup S_{2,2,2}$ or $C_{4} \cup C_{q}$ is an induced subgraph of $H^{\prime}$ and has two eigenvalues greater than 2 and cannot be an induced subgraph of $G$.

- If $y^{\prime} \neq y$ and $y^{\prime} \neq x$ then by the previous cases we necessarily have $\min \left\{d\left(y^{\prime}, u\right), d\left(y^{\prime}, v\right)\right\}=4$.

If $x^{\prime}$ is adjacent to another vertex $z$ in $H$ distinct from $y^{\prime}$ and $y$ then by the previous cases we necessarily have

$\min \{d(z, u), d(z, v)\}=4$ and either $S_{2,2,2} \cup S_{1,2,5}$ or $C_{r} \cup C_{s}$ is an an induced subgraph of $H^{\prime}$ and has two eigenvalues greater than 2 and cannot be an induced subgraph of $G$.

If $x^{\prime}$ is not adjacent to another vertex of $H$ then the graph $T_{n} \cup C_{q}$ or the graph $S_{1,3,3} \cup S_{1,3,3}$ is an induced subgraph of $H^{\prime}$ and has two eigenvalues greater than 2 and cannot be an induced subgraph of $G$.

* If $10 \leq b \leq c$ then $Q_{H}(2)>0$ and $H$ has two eigenvalues greater than 2 and cannot be an induced subgraph of $G$.

Second case: $p_{1}=4$.

We have $p_{2}=4$ and $p_{3} \in\{4,6,8\}$.

According to table $8, \lambda_{1}(P(4,4,4))>\sqrt{2+2 \sqrt{2}}$ and $P(4,4,4)$ cannot be an induced subgraph of a graph cospectral with $L(4, k)$ (table 11). When $p_{3} \in\{6,8\}$, $P\left(4,4, p_{3}\right)$ cannot be a connected component of a graph cospectral with $L(4, k)$ because $\lambda_{1}\left(P\left(4,4, p_{3}\right)\right)<2.1854$ while $\lambda_{1}(L(4, k)) \geq \lambda_{1}(L(4,3))>2.1888$ when $k \geq 3$. So there is a new vertex $x$ adjacent to one vertex $y$ of $P\left(4,4, p_{3}\right)$ (and only one because otherwise there exists $r, s \in \mathbb{N}$ such that $P(1, r, s)$ is an induced subgraph of $G$ which is impossible by lemma 5$)$. Let $H$ be the subgraph induced by $P\left(4,4, p_{3}\right)$ and $x$, these graphs $H$ are summed up in table 10 which shows that that $H$ cannot be an induced subgraph of $G$ because either $H$ has two eigenvalues greater than 2 or $H$ has a spectral radius greater than $\sqrt{2+2 \sqrt{2}}$. 


\section{A.3 Tables of some graphs eigenvalues}

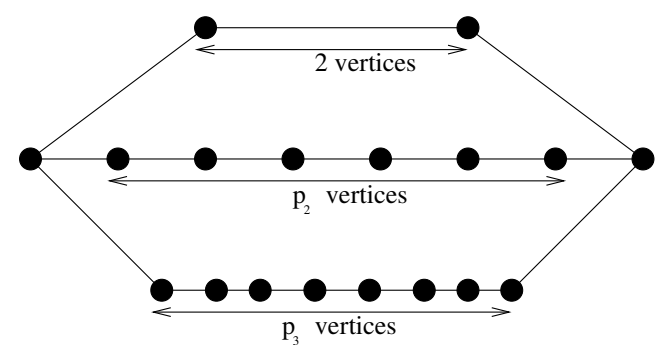

\begin{tabular}{|c||c|c|c|}
\hline \multicolumn{1}{|r||}{$p_{2}$} & 6 & 8 & 10 \\
\hline \hline \multirow{2}{*}{6} & 2.1987 & 2.1921 & 2.1891 \\
& 1.9122 & 1.9426 & 1.19604 \\
\hline \multirow{2}{*}{8} & 2.1921 & 2.1853 & 2.1822 \\
& 1.9426 & 1.9666 & 1.19805 \\
\hline \multirow{2}{*}{10} & 2.1891 & 2.1822 & 2.1790 \\
& 1.9604 & 1.9805 & 1.9922 \\
\hline \multirow{2}{*}{12} & 2.1878 & 2.1808 & 2.1776 \\
& 1.9716 & 1.9891 & 1.9994 \\
\hline \multirow{2}{*}{14} & 2.1872 & 2.1802 & 2.1770 \\
& 1.9790 & 1.9947 & 2.0041 \\
\hline \multirow{2}{*}{16} & 2.1870 & 2.1800 & 2.1767 \\
& 1.9842 & 1.9986 & 2.0072 \\
\hline \multirow{2}{*}{40} & 2.1868 & & \\
& 1.9999 & & \\
\hline
\end{tabular}

Table 6: The two largest eigenvalues of $P\left(2, p_{2}, p_{3}\right)$ with a 4 decimal place accuracy.

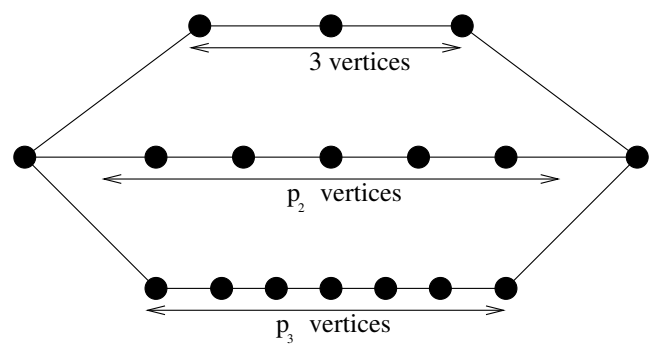

\begin{tabular}{|c|c|c|}
\hline$p_{3}$ & 5 & 7 \\
\hline \multirow{2}{*}{5} & 2.1940 & 2.1847 \\
\hline & 1.9319 & 1.9696 \\
\hline \multirow{2}{*}{7} & 2.1847 & 2.1753 \\
\hline & 1.9696 & 2.0000 \\
\hline \multirow{2}{*}{9} & 2.1804 & 2.1709 \\
\hline & 1.9890 & 2.0153 \\
\hline \multirow{2}{*}{11} & 2.1785 & 2.1689 \\
\hline & 2.0000 & 2.0237 \\
\hline
\end{tabular}

Table 7: The two largest eigenvalues of $P\left(3, p_{2}, p_{3}\right)$ with a 4 decimal place accuracy. 


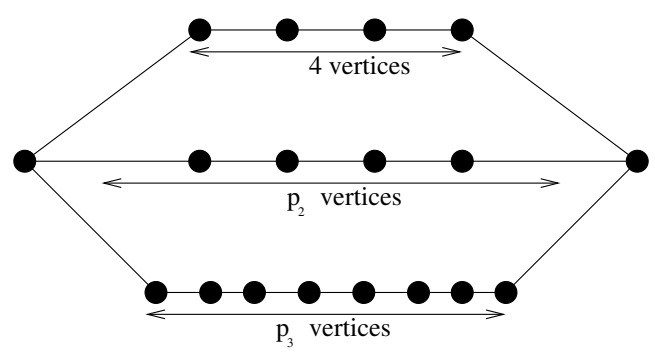

\begin{tabular}{|c|c|c|}
\hline$p_{3}$ & 4 & 6 \\
\hline \multirow{2}{*}{4} & 2.1987 & 2.1853 \\
\hline & 1.9122 & 1.9666 \\
\hline \multirow{2}{*}{6} & 2.1853 & 2.1723 \\
\hline & 1.9666 & 2.0102 \\
\hline \multirow{2}{*}{8} & 2.1790 & 2.1660 \\
\hline & 1.9922 & 2.0300 \\
\hline \multirow{2}{*}{10} & 2.1762 & 2.1631 \\
\hline & 2.0058 & 2.0401 \\
\hline
\end{tabular}

Table 8: The two largest eigenvalues of $P\left(4, p_{2}, p_{3}\right)$ with a 4 decimal place accuracy.

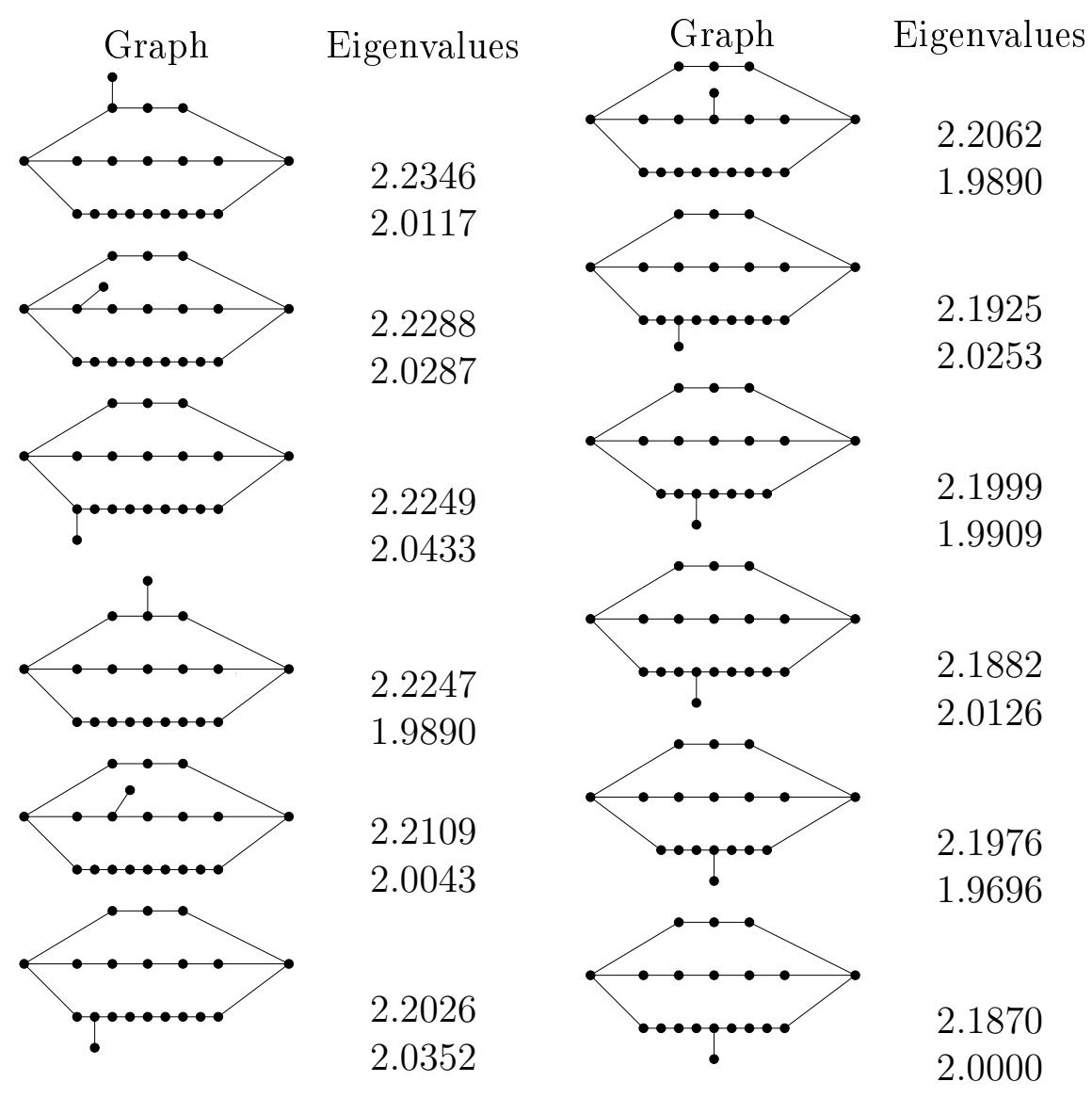

Table 9: The two largest eigenvalues of some graphs $H$ with a 4 decimal place accuracy. Note that the spectral radius increases when the number of vertices between two vertices of degree 3 decreases (theorem 7). 


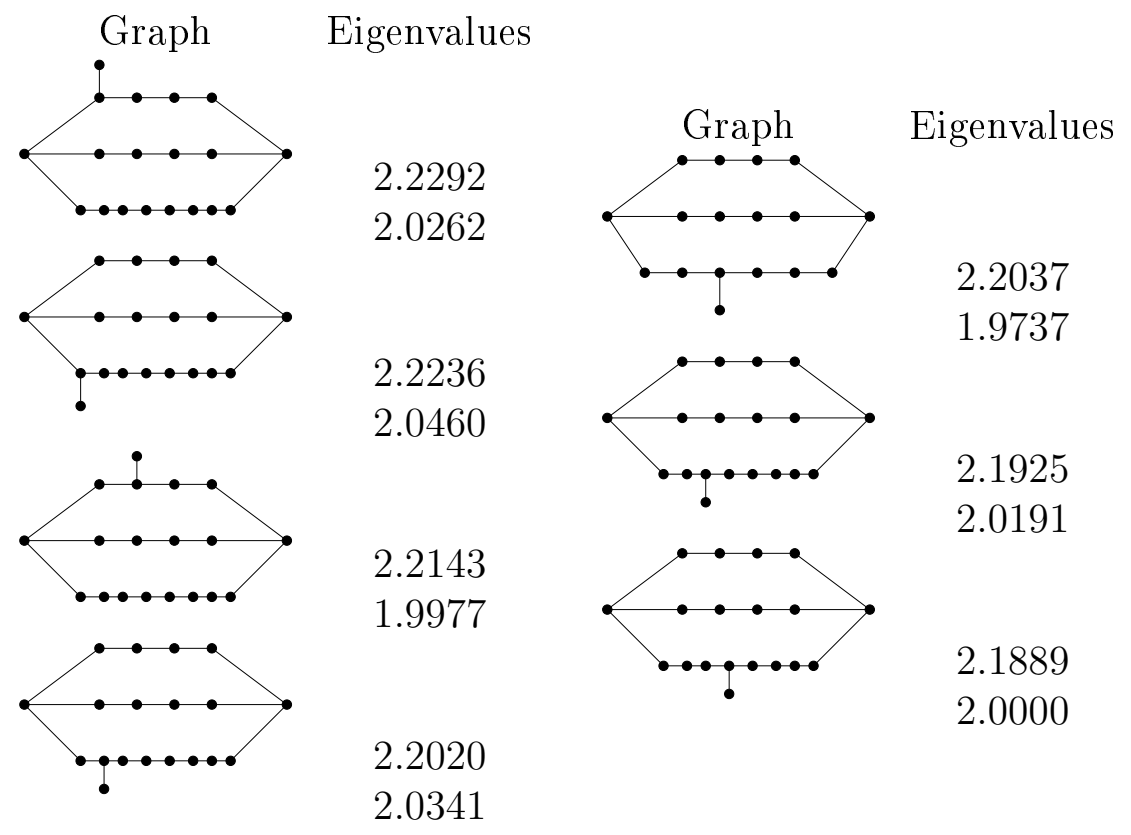

Table 10: The two largest eigenvalues of some graphs $H$ with a 4 decimal place accuracy. Note that the spectral radius increases when the number of vertices between two vertices of degre 3 decreases (theorem 7 ).

\begin{tabular}{|c|c|c|c|c|c|c|c|}
\hline$k$ & 1 & 2 & 3 & 4 & 5 & 6 & 7 \\
\hline$\lambda_{1}(L(4, k))$ & 2.1358 & 2.1753 & 2.1889 & 2.1940 & 2.1960 & 2.1968 & 2.1971 \\
\hline$k$ & 8 & 9 & 10 & 11 & 12 & 13 & 14 \\
\hline$\lambda_{1}(L(4, k))$ & 2.1973 & 2.1973 & 2.1974 & 2.1974 & 2.1974 & 2.1974 & 2.1974 \\
\hline
\end{tabular}

Table 11: Spectral radius of $L(4, k)$ with a 4 decimal place accuracy. 


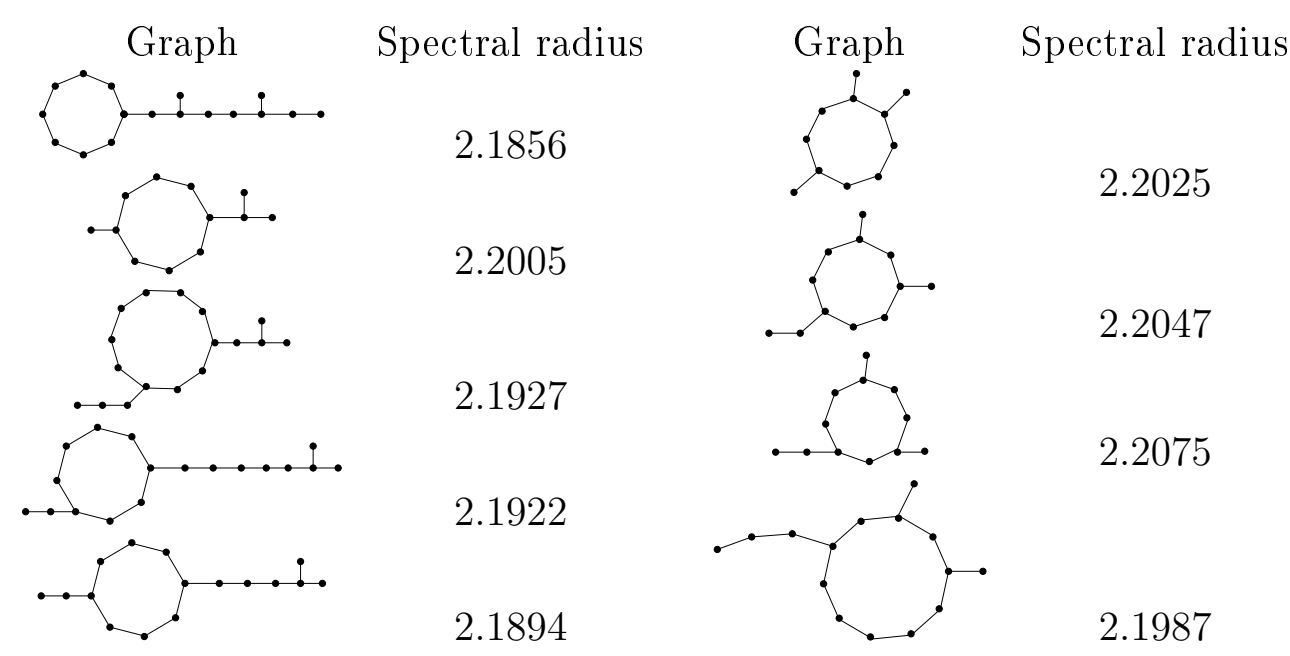

Table 12: Spectral radius of some unicyclic graphs with a 4 decimal place accuracy.

\section{References}

[1] D. Cvetkovic, M. Doob, and Saschs H. Spectra of graphs. Academic press, 1978.

[2] D. Cvetković and P. Rowlinson. Spectra of unicyclic graphs. Graphs and Combinatorics, 3:7-23, 1987.

[3] E. R. van Dam and W. H. Haemers. Developments on spectral characterizations of graphs. Discrete Mathematics, to appear.

[4] E.R. van Dam and W.H. Haemers. Which graphs are determined by their spectrum? Linear Algebra and its Applications, 373:241-272, 2003.

[5] M. Doob and W.H. Haemers. The complement of the path is determined by its spectrum. Linear Algebra and its Applications, 356:57-65, 2002.

[6] O. Favaron, M. Mahéo, and J.-F. Scalé. Some eigenvalues properties in graphs (conjectures of graffiti - ii). Discrete Mathematics, 111:197-200, 1993.

[7] C Godsil and G. Royle. Algebraic Graph Theory. Springer, 2001.

[8] W.H. Haemers, X. Liu, and Y. Zhang. Spectral characterizations of lollipop graphs. Linear Algebra and its Applications, 428:2405-3034, 2008.

[9] A.J. Hoffman and Smith J.H. On the spectral radii of topologically equivalent graphs. Recent Advances in Graph Theory, pages 273-281, 1975.

[10] X. Shen, Y. Hou, and Zhang Y. Graph $Z_{n}$ and some graphs related to $Z_{n}$ are determined by their spectrum. Linear Algebra and its Applications, 404:58-68, 2005.

[11] S.K. Simić and Kocic V.L. On the largest eigenvalue of some homeomorphic graphs. Publications de l'Institut Mathematique, Nouvelle série, 40:3-9, 1986.

[12] W. Wang and C.-X. Xu. On spectral characterisation of T-shape trees. Linear Algebra and its Applications, 414:492-501, 2006. 